\title{
Characterization of midostaurin as a dual inhibitor of FLT3 and SYK and potentiation of FLT3 inhibition against FLT3-ITD-driven leukemia harboring activated SYK kinase
}

\author{
Ellen L. Weisberg ${ }^{1,6}$, Alexandre Puissant ${ }^{2,6}$, Richard Stone ${ }^{1,6}$, Martin Sattler ${ }^{1,6}$, \\ Sara J. Buhrlage ${ }^{3,4,6}$, Jing Yang ${ }^{3,6}$, Paul W. Manley ${ }^{5}$ Chengcheng Meng ${ }^{1}$, Michael \\ Buonopane $^{1}$, John F. Daley ${ }^{1}$, Suzan Lazo ${ }^{1}$, Renee Wright ${ }^{1}$, David M. Weinstock ${ }^{1,6}$, \\ Amanda L. Christie ${ }^{1}$, Kimberly Stegmaier ${ }^{2,6}$ and James D. Griffin ${ }^{1,6}$ \\ ${ }^{1}$ Department of Medical Oncology, Dana-Farber Cancer Institute, Boston, Massachusetts, USA \\ ${ }^{2}$ Department of Pediatric Oncology, Dana-Farber Cancer Institute, Boston, Massachusetts, USA \\ ${ }^{3}$ Department of Cancer Biology, Dana-Farber Cancer Institute, Boston, Massachusetts, USA \\ ${ }^{4}$ Department of Biological Chemistry and Molecular Pharmacology, Boston, Massachusetts, USA \\ ${ }^{5}$ Novartis Institutes of Biomedical Research, Basel, Switzerland \\ ${ }^{6}$ Department of Medicine, Harvard Medical School, Boston, Massachusetts, USA \\ Correspondence to: Ellen L. Weisberg, email: ellen_weisberg@dfci.harvard.edu
}

James D. Griffin, email: james_griffin@dfci.harvard.edu

Keywords: acute myelogenous leukemia, midostaurin, R406, R788, FLT3-ITD

Received: March 16, $2017 \quad$ Accepted: May 12, $2017 \quad$ Published: July 06, 2017

Copyright: Weisberg et al. This is an open-access article distributed under the terms of the Creative Commons Attribution License 3.0 (CC BY 3.0), which permits unrestricted use, distribution, and reproduction in any medium, provided the original author and source are credited.

\section{ABSTRACT}

Oncogenic FLT3 kinase is a clinically validated target in acute myeloid leukemia (AML), and both multi-targeted and selective FLT3 inhibitors have been developed. Spleen tyrosine kinase (SYK) has been shown to be activated and increased in FLT3ITD-positive AML patients, and has further been shown to be critical for transformation and maintenance of the leukemic clone in these patients. Further, over-expression of constitutively activated SYK causes resistance to highly selective FLT3 tyrosine kinase inhibitors (TKI). Up to now, the activity of the multi-targeted FLT3 inhibitor, midostaurin, against cells expressing activated SYK has not been explored in the context of leukemia, although SYK has been identified as a target of midostaurin in systemic mastocytosis. We compared the ability of midostaurin to inhibit activated SYK in mutant FLT3-positive AML cells with that of inhibitors displaying dual SYK/ FLT3 inhibition, targeted SYK inhibition, and targeted FLT3 inhibition. Our findings suggest that dual FLT3/SYK inhibitors and FLT3-targeted drugs potently kill oncogenic FLT3-transformed cells, while SYK-targeted small molecule inhibition displays minimal activity. However, midostaurin and other dual FLT3/SYK inhibitors display superior anti-proliferative activity when compared to targeted FLT3 inhibitors, such as crenolanib and quizartinib, against cells co-expressing FLT3-ITD and constitutively activated SYK-TEL. Interestingly, additional SYK suppression potentiated the effects of dual FLT3/SYK inhibitors and targeted FLT3 inhibitors against FLT3-ITD-driven leukemia, both in the absence and presence of activated SYK. Taken together, our findings have important implications for the design of drug combination studies in mutant FLT3-positive patients and for the design of future generations of FLT3 inhibitors. 


\section{INTRODUCTION}

Around $30 \%$ of patients with acute myeloid leukemia (AML) harbor activating mutations in FLT3 [1], a gene normally involved in regulating hematopoiesis. The most common type of FLT3 mutation results in internal tandem duplications (ITD) within the juxtamembrane domain, occurring in $20-25 \%$ of AML and strongly associated with decreased survival [2,3]. An additional $7 \%$ of patients have point mutations within the "activation loop" of FLT3 [4].

Numerous FLT3 kinase inhibitors, both multitargeted and selective, have been developed [5]. The $\mathrm{N}$-indolocarbazole, midostaurin (PKC412; N-benzoylstaurosporine; Novartis Pharma AG) was shown to target oncogenic FLT3 in preclinical studies [6] was reported to significantly prolong survival of FLT3-mutated AML patients when combined with conventional induction and consolidation therapies in a randomized Phase III clinical trial [7]. Midostaurin was recently FDA approved for treatment of adult, newly diagnosed AML patients positive for oncogenic FLT3, in combination with standard chemotherapy. Other FLT3 inhibitors in clinical development include quizartinib (AC220), which exhibits high potency and selectivity against FLT3-ITD [8], and crenolanib besylate (CP-868596; AROG Pharmaceuticals, LLC) [9].

Spleen tyrosine kinase (SYK) is an obligatory signaling partner for FLT3 that is required for transformation to AML and necessary for myeloproliferative disease (MPD) development [10]. SYK (wild-type (wt)) is expressed in most hematopoietic cells $[11,12]$ and belongs to the SYK/ZAP-70 family of non-receptor tyrosine kinases $[13,14]$. Oncogenic SYK has been identified as an important driver of different hematologic malignancies, including B-cell lymphoma, chronic lymphocytic leukemia (CLL) and mantle cell lymphoma [15-17], and was identified as a target in AML with SYK inhibition exhibiting anti-leukemia activity in mouse models of AML [18]. ITK-SYK, which results from the fusion between SYK and ITK (IL-2-inducible T-cell kinase), occurs as a recurrent translocation in 17\% of patients with unspecified peripheral T-cell lymphomas $[19,20]$. TEL-SYK was originally detected in a patient with an atypical myelodysplastic syndrome with leukemic transformation $[21,22]$. TEL-SYK over-expression in murine pre B cells causes a B-acute lymphocytic leukemia (ALL)-like disease in mice [23] and cytoplasmic TEL-SYK fusion induces an acute panmyelosis with myelofibrosis-type acute myeloid leukemia (AML) in a bone marrow transplantation model [24].

Importantly, highly activated SYK has been found to be enriched in AML patients with a higher frequency in patients harboring the ITD mutant than wild-type (wt) FLT3 [10]. SYK has also been shown to be associated with resistance to FLT3-ITD-targeted therapy, and inhibition of
FLT3-ITD with quizartinib combined with inhibition of SYK was shown to be more effective than FLT3 inhibition alone in FLT3-ITD-positive models of AML [10].

Given the reported significance of SYK in transformation and maintenance of AML, as well as FLT3 kinase inhibitor resistance, and implications of SYK as a potentially important target for AML treatment, we were interested in investigating the ability of midostaurin to inhibit SYK in FLT3 mutant-positive AML. SYK has previously been shown to be a target of midostaurin and its metabolites, CGP52421 and CGP62221, in advanced systemic mastocytosis, a hematopoietic neoplasm characterized by expansion and abnormal accumulation of mast cells [25]. Up to now, however, the activity of midostaurin, alone and combined with SYK inhibition, against cells expressing activated SYK has not been explored in the context of leukemia.

Here, we compare the SYK-targeting activity of midostaurin with different classes of kinase inhibitors, including those with SYK inhibitory activity and those without. We show that midostaurin, like the dual FLT3/SYK inhibitors, R406 (tamatinib), and R788 (fostamatinib) [26], inhibits SYK in cell-based models of FLT3-ITD- and activated SYK-driven leukemia to a greater extent than highly targeted inhibitors of FLT3. We also show that SYK inhibition alone is not sufficient to kill FLT3-ITD-positive cells and that the SYK inhibitory activity of midostaurin is insufficient to potently kill cells expressing activated SYK. However, additional targeted SYK inhibition or dual FLT3/SYK inhibition potentiates the effects of midostaurin and other inhibitors of FLT3 against both kinase inhibitor-sensitive- and -resistant FLT3-ITD- and activated SYK-positive leukemia.

\section{RESULTS}

\section{SYK is a target of midostaurin}

As a first assessment of the ability of midostaurin to inhibit SYK we tested its inhibitory activity of SYK in a purified enzyme assay and found the compound inhibits SYK with an $\mathrm{IC}_{50}$ of $20.8 \mathrm{nM}$. In similar enzyme assays, the SYK inhibitor, R788, and its active metabolite, R406, have been determined to inhibit SYK with an $\mathrm{IC}_{50}$ of 41 $\mathrm{nM}$ [26]. Both compounds are reported to be 5-fold less potent against FLT3 than Syk. Both R788 and R406 have been evaluated in clinical trials. In comparison, a highly targeted SYK inhibitor, PRT062607 (P505, BIIB057) has been reported in cell-free assays to inhibit SYK with an $\mathrm{IC}_{50}$ of $1 \mathrm{nM}$ and to inhibit FLT3 with an $\mathrm{IC}_{50}$ of $139 \mathrm{nM}$ [27]. 


\section{Effects of midostaurin, R406, and $\mathrm{R788}$ on $\mathrm{Ba} / \mathrm{F} 3$ cells expressing activated SYK}

In order to investigate the ability of midostaurin to inhibit SYK activity in cells, we utilized $\mathrm{Ba} / \mathrm{F} 3$ cell lines that stably express constructs encoding fusion proteins comprised of SYK kinase coupled with a TEL moiety that constitutively activates SYK [10] (Supplementary Figure 1). Specifically, one Ba/F3 cell line was developed to overexpress constitutively active "TEL-SYK," the protein product of a fusion between a truncated form of $S Y K$ that is devoid of its SH2 domains, $\mathrm{SH} 2 \mathrm{Nter}+\mathrm{SH} 2 \mathrm{Cter}$, and a TEL sequence that takes the place of these two domains $[22,10]$. A second $\mathrm{Ba} / \mathrm{F} 3$ cell line tested was engineered to over-express a constitutively and highly active variant of SYK, "SYK-TEL," which, unlike TEL-SYK, contains an intact SH2 domain (Supplementary Figure 1). Consistent with the order of potencies generated in cell-free assays, midostaurin inhibited the growth of Ba/F3-TEL-SYK cells with an $\mathrm{IC}_{50}$ of $101.2 \mathrm{nM}$, a 2- to 3-fold higher potency than that of $\mathrm{R} 406\left(\mathrm{IC}_{50}=196.8 \mathrm{nM}\right)$ and $\mathrm{R} 788\left(\mathrm{IC}_{50}=\right.$ $332.9 \mathrm{nM}$ ), however a lower potency than the $\mathrm{IC}_{50}$ of $43.8 \mathrm{nM}$ for PRT062607 against these cells (Figure 1A1D and Table 1A). The effects of midostaurin, R406, and $\mathrm{R} 788$ against $\mathrm{Ba} / \mathrm{F} 3-\mathrm{TEL}-\mathrm{SYK}$ cells were modestly IL-3 rescued; the effects of PRT062607 were highly IL-3 rescue-able.

Similar results were observed with midostaurin treatment of $\mathrm{Ba} / \mathrm{F} 3-\mathrm{SYK}$-TEL cells, with a higher potency exhibited by midostaurin $\left(\mathrm{IC}_{50}=108.0 \mathrm{nM}\right)$ as compared to R406 $\left(\mathrm{IC}_{50}=142.4 \mathrm{nM}\right)$ and $\mathrm{R} 788\left(\mathrm{IC}_{50}=237.7 \mathrm{nM}\right)$, yet a lower potency as compared to PRT062607 $\left(\mathrm{IC}_{50}=\right.$ $30.0 \mathrm{nM}$ ) (Figure 1E-1H and Table 1A). Midostaurin was observed to induce apoptosis, as measured by annexin/ PI staining, in cells expressing constitutively activated
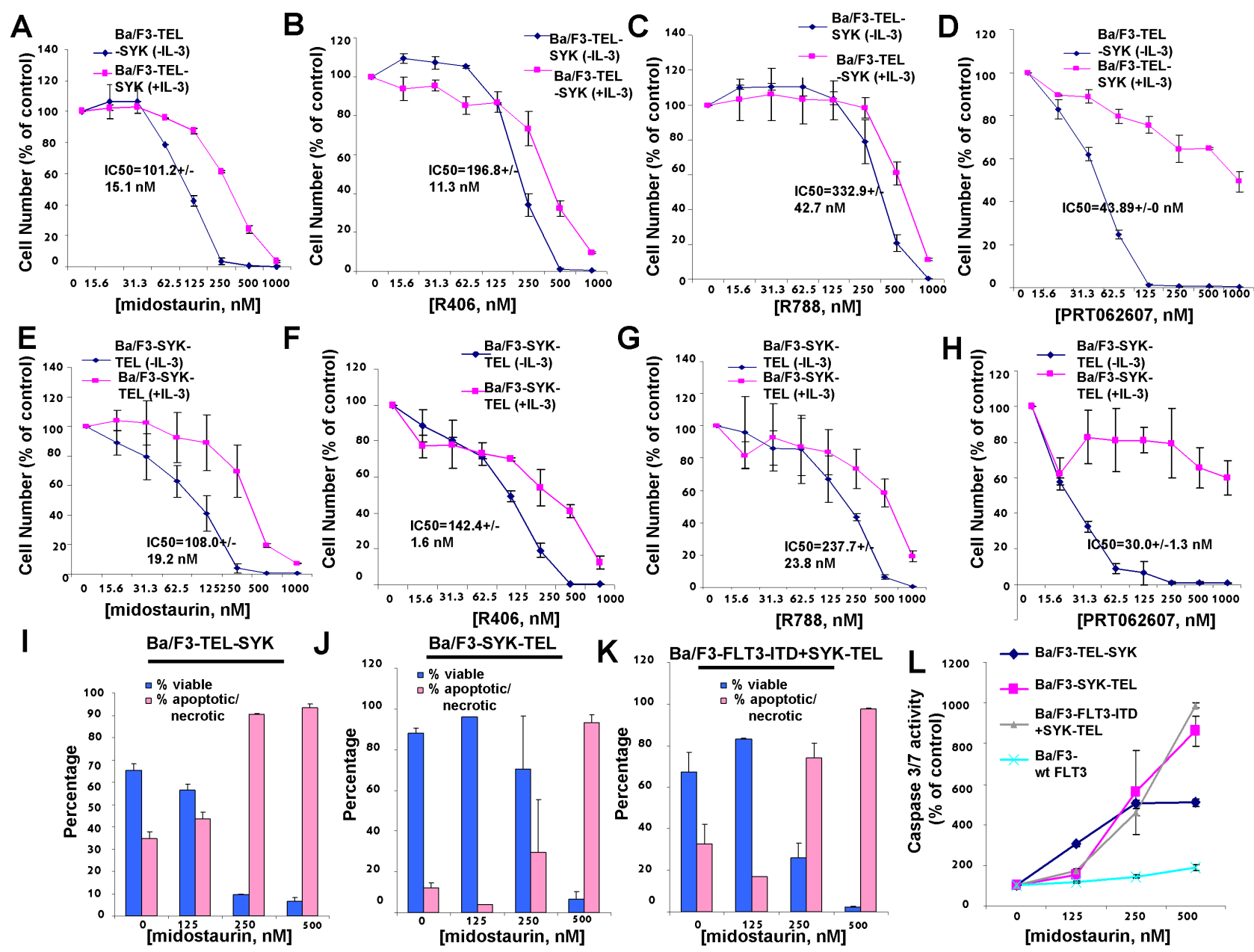

Figure 1: Effects of midostaurin, R406, R788, and PRT062607 on inhibition of proliferation of $B a / F 3$ cells expressing constitutively activated TEL-SYK or constitutively activated SYK-TEL. A.-D. Three-day treatment of Ba/F3-TEL-SYK cells with midostaurin (A), R406 (B), R788 (C), or PRT062607 (D) in the presence and absence of 20\% WEHI (used as a source of IL-3). E.H. Three-day treatment of Ba/F3-SYK-TEL cells with midostaurin (E), R406 (F), R788 (G), or PRT062607 (H). Error bars represent the mean+/-S.D. for all proliferation studies. I.-K. Effects of midostaurin on apoptosis (annexin/pi measurement) in cells expressing active SYK. Error bars represent the mean+/-S.D. L. Effects of midostaurin on apoptosis (caspase 3/7 measurement) in cells expressing active SYK. Error bars represent the mean+/-S.D. 
Table 1A: IC50s (nM) (mean+/-S.D.), generated using GraphPad Prism 7 Software, for R406, midostaurin, R788, and PRT062607 tested against Ba/F3-SYK-TEL, Ba/F3-TEL-SYK, Ba/F3-FLT3-ITD, Ba/F3-FLT3-ITD+SYK-TEL, and Ba/F3-FLT3-ITD+TEL-SYK. Cell growth assays were carried out for approximately 3 days.

\begin{tabular}{|l|c|c|c|c|}
\hline & $\begin{array}{c}\text { R406 } \\
(\mathbf{I C 5 0}, \mathbf{n M})\end{array}$ & $\begin{array}{c}\text { midostaurin } \\
(\mathbf{I C 5 0}, \mathbf{n M})\end{array}$ & $\begin{array}{c}\text { R788 } \\
(\mathbf{I C 5 0 ,} \mathbf{n M})\end{array}$ & $\begin{array}{c}\text { PRT062607 } \\
(\mathbf{I C 5 0 ,} \mathbf{n M})\end{array}$ \\
\hline Ba/F3-SYK-TEL & $142.4+/-1.6$ & $108.0+/-19.2$ & $237.7+/-23.8$ & $30.0+/-1.3$ \\
\hline Ba/F3-TEL-SYK & $196.8+/-11.3$ & $101.2+/-15.1$ & $332.9+/-42.7$ & $43.8+/-0.1$ \\
\hline Ba/F3-FLT3-ITD & $36.7+/-20.5$ & $6.3+/-3$ & $61.5+/-18.9$ & $1078.0+/-110.4$ \\
\hline Ba/F3-FLT3-ITD+SYK-TEL & $223.7+/-42.2$ & $198.2+/-21.6$ & $347.8+/-71.4$ & $48.4+/-0.3$ \\
\hline Ba/F3-FLT3-ITD+TEL-SYK & $27.0+/-3.4$ & $3.0+/-0.2$ & $47.1+/-6.3$ & 614.4 \\
\hline
\end{tabular}

SYK (Figure 1I-1K). Consistent with this, midostaurin also more robustly increased the activities of caspase-3 and -7 in activated SYK-expressing cells as compared to growth factor-dependent $\mathrm{Ba} / \mathrm{F} 3$ cells over-expressing wt FLT3 (Figure 1L) and led to an increase in the subG0/ G1 fraction as determined by PI staining (Supplementary Figure 2).

\section{Effects of midostaurin, R406, and R788 on Ba/F3 cells co-expressing FLT3-ITD and activated SYK}

Oncogenic FLT3 has been previously demonstrated to be phosphorylated by constitutively activated SYK through its SH2 domain, and co-expression of SYKTEL and FLT3-ITD leads to an association between the two proteins and renders cells resistant to FLT3 kinase inhibition [10]. We were interested in comparing the activities of dual FLT3/SYK inhibitors, midostaurin, R406, and R788, and the selective SYK inhibitor, PRT062607, against Ba/F3-FLT3-ITD+SYK-TEL cells. Compared to drug effects on $\mathrm{Ba} / \mathrm{F} 3-\mathrm{SYK}$-TEL cells, a 1.51.8-fold loss of potency against Ba/F3-FLT3-ITD+SYKTEL cells was observed for midostaurin (IC50 $=198.2$ $\mathrm{nM}$ ), R406 (IC50=223.7 nM), and R788 (IC50=347.8 $\mathrm{nM}$ ), and PRT062607 (IC50=48.4 nM) (Figure 1E$1 \mathrm{H}$, Figure 2A-2D, and Table 1A). Inhibition of growth of cells co-expressing FLT3-ITD and SYK-TEL by midostaurin correlated with induction of apoptosis (Figure $1 \mathrm{~K})$. Potency differences for the inhibitors against $\mathrm{Ba} /$ F3-SYK-TEL versus Ba/F3-FLT3-ITD+SYK-TEL cells were reflected in the ability of midostaurin and R406 to suppress the activity of signaling molecules downstream of SYK and FLT3 to a slightly higher extent in $\mathrm{Ba} / \mathrm{F} 3$ SYK-TEL cells than Ba/F3-FLT3-ITD+SYK-TEL cells, including STAT5, AKT, and S6 (Supplementary Figure 3A-3B). Similarly, inhibition of SYK phosphorylation by PRT062607 in Ba/F3-SYK-TEL cells was more significant than in Ba/F3-FLT3-ITD+SYK-TEL cells (Figure 2E).

The SH2-binding region expressed on SYK-TEL but missing from TEL-SYK allows phosphorylation of
FLT3 by SYK through a functional interaction between SYK and FLT3 [10] (Supplementary Figure 1). TELSYK, in contrast, despite being constitutively active, is not able to activate FLT3 to the extent of SYK-TEL [10] (Supplementary Figure 1). Midostaurin, R406, and R788 displayed high potencies against Ba/F3-FLT3ITD+TEL-SYK cells, with IC50 values of $3.0 \mathrm{nM}, 27$ $\mathrm{nM}$, and $47.1 \mathrm{nM}$, respectively (Figure $2 \mathrm{~F}-2 \mathrm{H}$ and Table 1A), as compared to Ba/F3-FLT3-ITD+SYK-TEL cells. PRT062607, in contrast, displayed comparatively low potency against $\mathrm{Ba} / \mathrm{F} 3-\mathrm{FLT3}$-ITD+TEL-SYK cells (IC50 $614.4 \mathrm{nM}$ ) (Figure 2I). Thus, although this line was included for investigation, the data suggest that it may be predominantly driven by FLT3-ITD. In support of this, the potencies of midostaurin, R406, R788, and PRT062607 against Ba/F3-FLT3-ITD+TEL-SYK were very similar to potencies of these compounds against Ba/F3-FLT3-ITD cells, with IC50 values of $6.3 \mathrm{nM}, 36.7 \mathrm{nM}, 61.5 \mathrm{nM}$, and $1078 \mathrm{nM}$, respectively (Figure 3A-3D and Table 1A).

For comparison with $\mathrm{Ba} / \mathrm{F} 3$ cells expressing FLT3ITD, we tested the effects of dual SYK/FLT3 and targeted SYK inhibitors against cells expressing FLT3-ITD and kinase inhibitor-resistant tyrosine kinase domain (TKD) point mutations. As expected, midostaurin, R406, and R788 treatment killed Ba/F3 cells expressing FLT3ITD+FLT3 kinase inhibitor-resistant TKD point mutants to varying extents although each generally with less potency than toward FLT3-ITD, whereas PRT062607 was completely inactive against these cells (Figure 3E-3H).

We next explored the ability of midostaurin, R406, and PRT062607 to inhibit the phosphorylation of FLT3 or $\mathrm{SYK}$ in $\mathrm{Ba} / \mathrm{F} 3$ cells transformed by activated versions of each. Midostaurin and R406 were effective at inhibiting FLT3 phosphorylation in a concentration-dependent manner in $\mathrm{Ba} / \mathrm{F} 3-\mathrm{FLT} 3-\mathrm{ITD}$ cells, which correlated with induction of cell death by these compounds (Figure 3I3J). In Ba/F3-TEL-SYK cells, midostaurin, similar to PRT062607, inhibited TEL-SYK phosphorylation and activity of downstream effectors of TEL-SYK across a concentration range that led to inhibition of cell growth by the compounds (Figure 4A). In Ba/F3-FLT3-ITD+TEL- 
SYK cells, both midostaurin and R406 inhibited phosphorylation of TEL-SYK, although to a lesser extent than PRT062607 (Figure 4B-4D). Despite the observed inhibitory activity of PRT062607 against TEL-SYK, the fact that PRT062607 is a comparatively weak inhibitor of $\mathrm{Ba} / \mathrm{F} 3-F L T 3-I T D+T E L-S Y K$ proliferation again supports the notion that Ba/F3-FLT3-ITD+TEL-SYK cells are predominantly driven by FLT3-ITD.

Midostaurin, R406, R788, quizartinib, and crenolanib were tested in parallel against parental $\mathrm{Ba} /$ F3 cells (as a control for nonspecific toxicity), Ba/F3FLT3-ITD, and Ba/F3-FLT3-ITD+SYK-TEL to compare the anti-proliferative effects of inhibitors targeting both FLT3-ITD and SYK (midostaurin, R406, R788) and those targeting FLT3-ITD alone (quizartinib, crenolanib) (Figure 5). Compared to $\mathrm{IC}_{50} \mathrm{~s}$ generated for midostaurin (6.3
$\mathrm{nM})$, R406 (36.7nM), and R788 (61.5 nM) against $\mathrm{Ba}$ / F3-FLT3-ITD cells, treatment of Ba/F3-FLT3-ITD+SYKTEL cells with midostaurin (198.2 nM), R406 (223.7 nM), or R788 (347.8 nM) led to an average 31.5-fold shift for midostaurin, 6.1-fold shift for R406, and 5.7-fold shift for R788 (Figure 5A-5C). In contrast, compared to the $\mathrm{IC}_{50} \mathrm{~S}$ for quizartinib $(0.3 \mathrm{nM})$ or crenolanib $(6.2 \mathrm{nM})$ against $\mathrm{Ba}$ / F3-FLT3-ITD cells, treatment of Ba/F3-FLT3-ITD+SYKTEL cells with quizartinib $(627.5 \mathrm{nM})$ or crenolanib (520.6 $\mathrm{nM}$ ) led to an average 2092-fold higher $\mathrm{IC}_{50}$ for quizartinib and an 84-fold higher for crenolanib (Figure 5D-5E). Graphing of drug effects against Ba/F3-FLT3-ITD+SYKTEL cells together shows less responsiveness of these cells to targeted FLT3 inhibitors, such as crenolanib and quizartinib, both of which lack SYK as a target, compared to midostaurin, R788, and R406 (Figure 5F). These

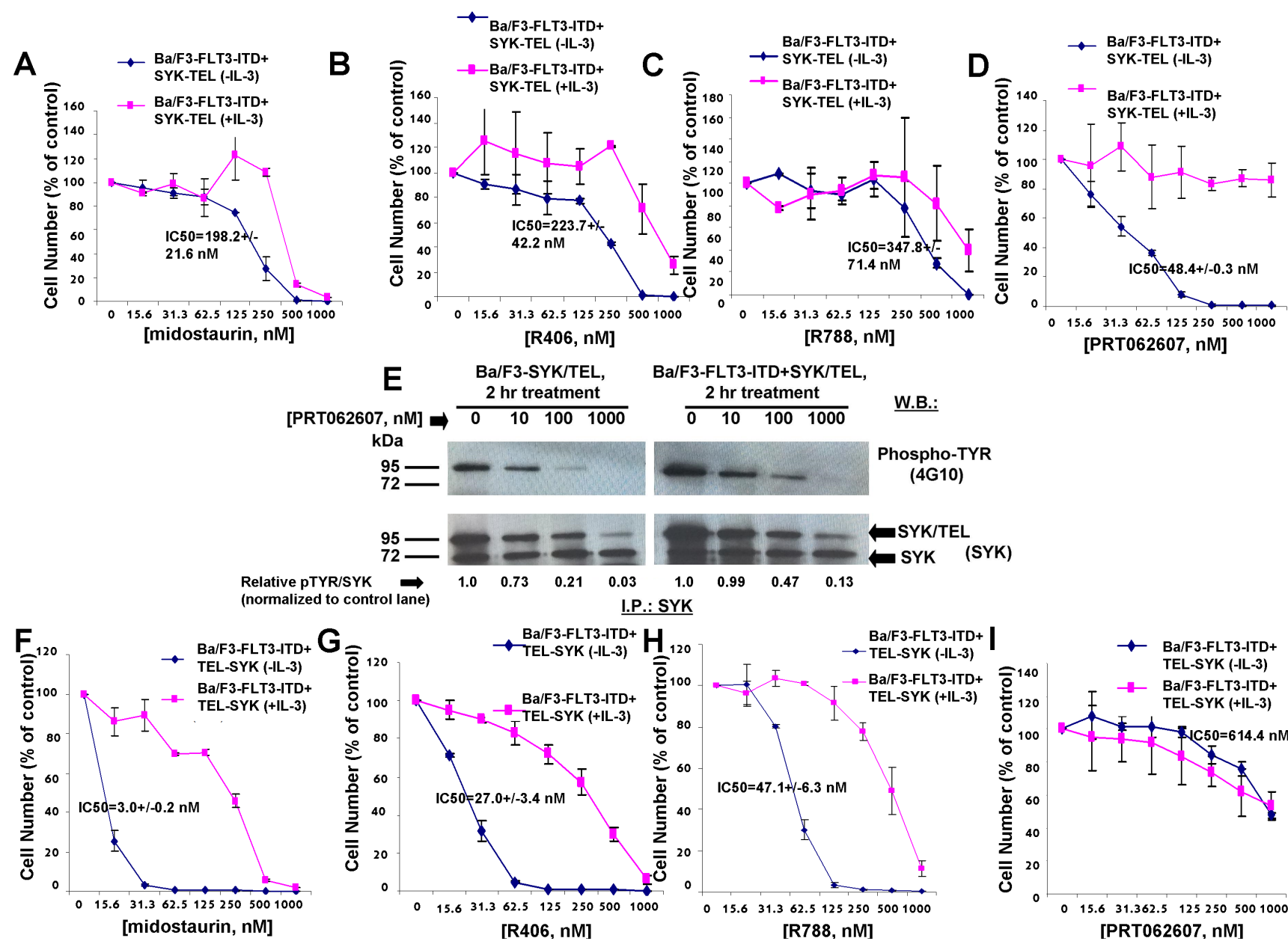

Figure 2: Effects of midostaurin, R406, R788, and PRT062607 on inhibition of proliferation of Ba/F3 cells expressing TEL-SYK or SYK-TEL co-expressed with FLT3-ITD. A.-D. Approximately 3-day treatment of Ba/F3-FLT3-ITD+SYK-TEL cells with midostaurin (A), R406 (B), R788 (C), or PRT062607 (D) in the presence and absence of 20\% WEHI (used as a source of IL-3). Error bars represent the mean+/-S.D. for all proliferation studies. E. Effect of PRT062607 on phosphorylation of SYK-TEL in Ba/F3-SYKTEL and Ba/F3-FLT3-ITD+SYK-TEL cells. For this experiment, SYK immunoprecipitation was carried out prior to immunoblotting with a PTYR (4G10) antibody. ImageJ 32 software was used for densitometry. Briefly, to get band intensities, the area of all the bands was first measured, and then normalized to the control lane, and then normalized to total protein. F.-I. Approximately 3-day treatment of Ba/ F3-FLT3-ITD+TEL-SYK cells with midostaurin (F), R406 (G), R788 (H), or PRT062607(I). Error bars represent the mean+/-S.D. for all proliferation studies. 
Table 1B: IC50s (nM) (mean+/-S.D.), generated using GraphPad Prism 7 Software, for crenolanib and AC220 tested against Ba/F3-FLT3-ITD and Ba/F3-FLT3-ITD+SYK-TEL. Cell growth assays were carried out for approximately 3 days.

\begin{tabular}{|l|c|c|}
\hline & $\begin{array}{c}\text { crenolanib } \\
(\mathbf{I C 5 0}, \mathbf{n M})\end{array}$ & $\begin{array}{c}\text { quizartinib } \\
\text { (IC50, } \mathbf{n M})\end{array}$ \\
\hline $\mathrm{Ba} /$ F3-FLT3-ITD & $6.2+/-2$ & $0.3+/-0.3$ \\
\hline $\mathrm{Ba} /$ F3-FLT3-ITD+SYK-TEL & $520.6+/-121.1$ & $627.5+/-32.2$ \\
\hline
\end{tabular}

results suggest the importance of a FLT3 kinase inhibitor also possessing SYK inhibitory activity in order to more potently kill leukemia cells characterized as expressing both mutant FLT3 and activated SYK.

It is important to note that concentrations of R406, and $\mathrm{R} 788$ were relatively ineffective against parental $\mathrm{Ba} /$ F3 cells at up to 500-1000 nM following three days of drug treatment, however concentrations of midostaurin over $250 \mathrm{nM}$ led to anti-proliferative effects against parental $\mathrm{Ba} / \mathrm{F} 3$ cells suggesting that nonspecific toxicity could occur at those levels of drug (Figure 5). In contrast, PRT062607 was ineffective against parental $\mathrm{Ba} / \mathrm{F} 3$ cells at up to $1000 \mathrm{nM}$ (Supplementary Figure 4).
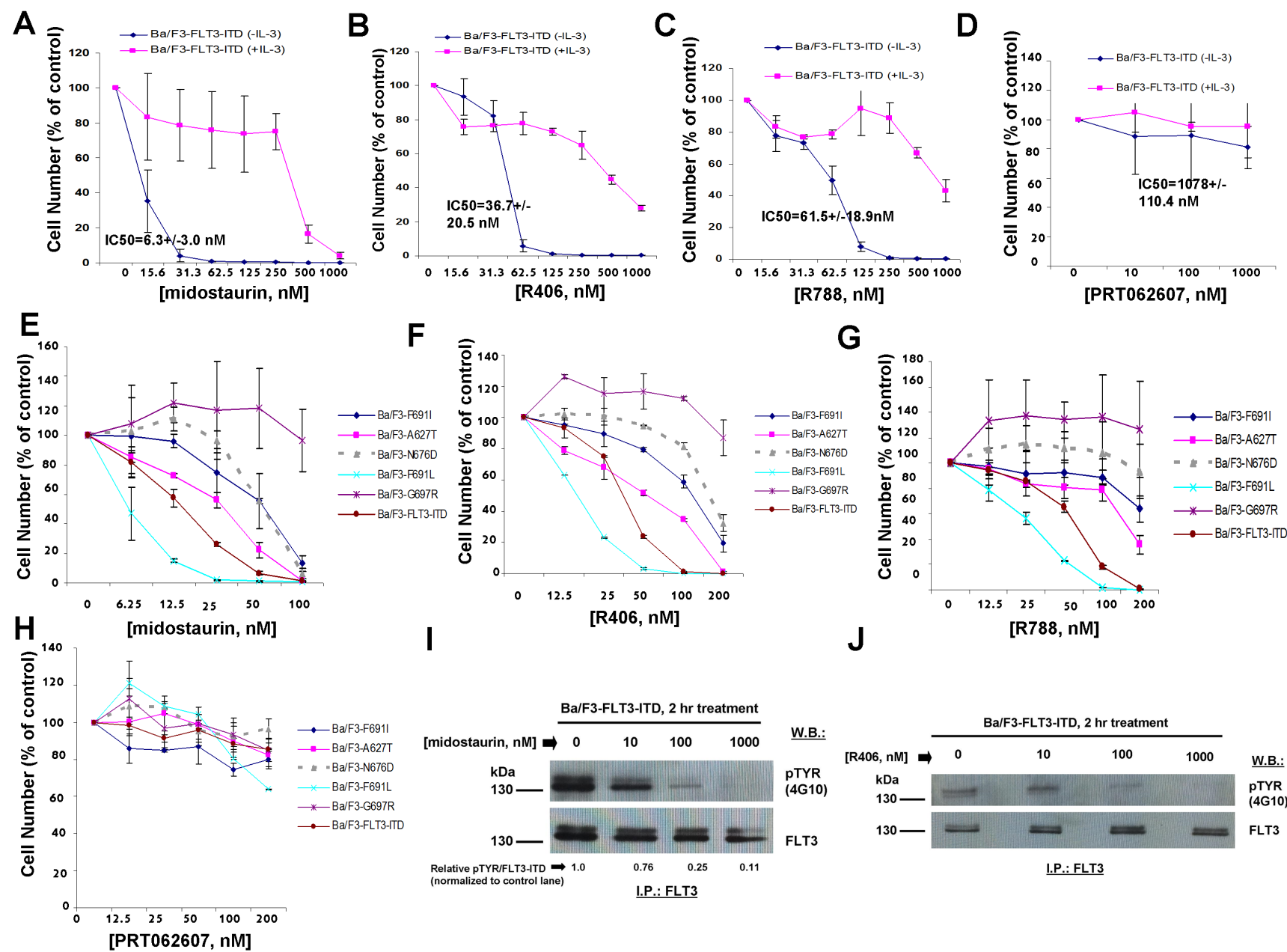

Figure 3: Effects of midostaurin, R406, R788, and PRT062607 on Ba/F3 cells expressing FLT3-ITD. A.-D. Three-day treatment of Ba/F3-FLT3-ITD cells with midostaurin (A), R406 (B), R788 (C), or PRT062607 in the presence and absence of 20\% WEHI (used as a source of IL-3). E.-H. Three-day treatment of kinase inhibitor-resistant FLT3-ITD+TKD point mutants with midostaurin (E), R406 (F), R788 (G), or PRT062607 (H). Error bars represent the mean+/-S.D. for all proliferation studies. I.-J. Effect of midostaurin (I) and R406 (J) on phosphorylation of FLT3-ITD. For this experiment, FLT3 immunoprecipitation was carried out prior to immunoblotting with a PTYR (4G10) antibody. ImageJ 32 software was used for densitometry. Briefly, to get band intensities, the area of all the bands was first measured, and then normalized to the control lane, and then normalized to total protein. 
Potentiation of the anti-leukemic effects of midostaurin by dual FLT3/SYK suppression and targeted SYK suppression

The ability of midostaurin to synergize with dual FLT3/SYK inhibitors, R406 and R788, or the highly targeted SYK inhibitor, PRT062607, was tested in a panel of $\mathrm{Ba} / \mathrm{F} 3$ cell lines expressing constitutively active $\mathrm{SYK}$ in the presence or absence of FLT3-ITD, as well as Ba/F3FLT3-ITD cells and human AML lines, such as MOLM13luc+, MOLM14, and MV4,11, driven by FLT3-ITD. As described in detail below, general observations from these experiments include potentiation of the anti-proliferative effects of midostaurin by inhibitors targeting SYK, which supports the notion that additional SYK suppression is

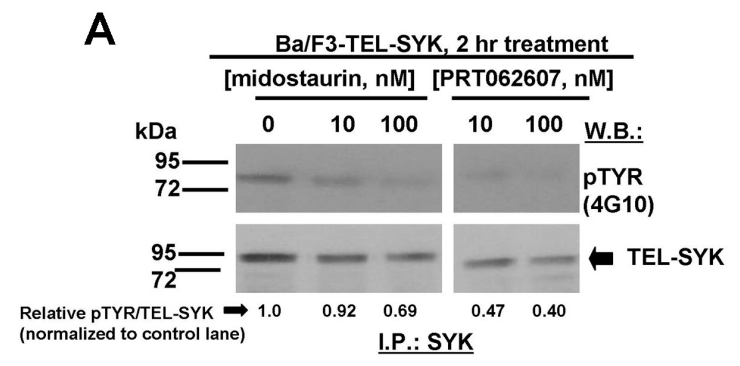

(SYK)

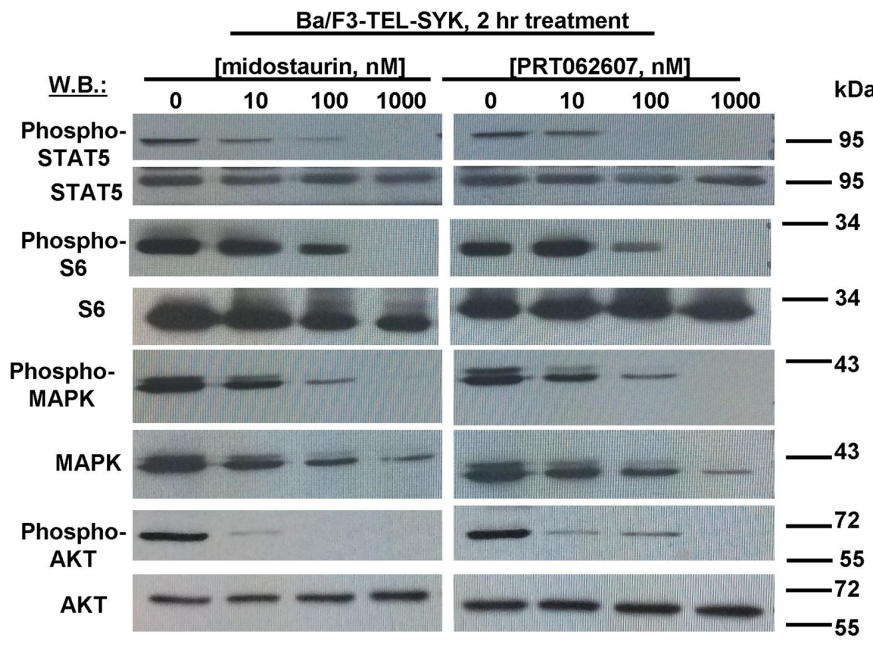

of benefit against FLT3-ITD-driven AML treated with a FLT3 kinase inhibitor.

Concentration ranges tested for the different compounds alone and in combination were based on their estimated cellular $\mathrm{IC}_{50}$ values. Effects of midostaurin against $\mathrm{Ba} / \mathrm{F} 3-\mathrm{TEL}-\mathrm{SYK}, \mathrm{Ba} / \mathrm{F} 3-\mathrm{SYK}-\mathrm{TEL}, \mathrm{Ba} / \mathrm{F} 3-$ FLT3-ITD+TEL-SYK, and Ba/F3-FLT3-ITD+SYK-TEL were potentiated to varying extents by R788, R406 and PRT062607, with additive to synergistic interactions observed (Figures 6A-6C, 7A-7C, 8A-8C, Supplementary Figure 5 and Table 2). Combination effects of midostaurin+/-R406 and midostaurin+/-PRT062607 that led to increased inhibition of proliferation of constitutively activated SYK-expressing cells correlated with increased suppression of phosphorylation of downstream effectors of SYK, including STAT5, S6, MAPK, and AKT (Figures
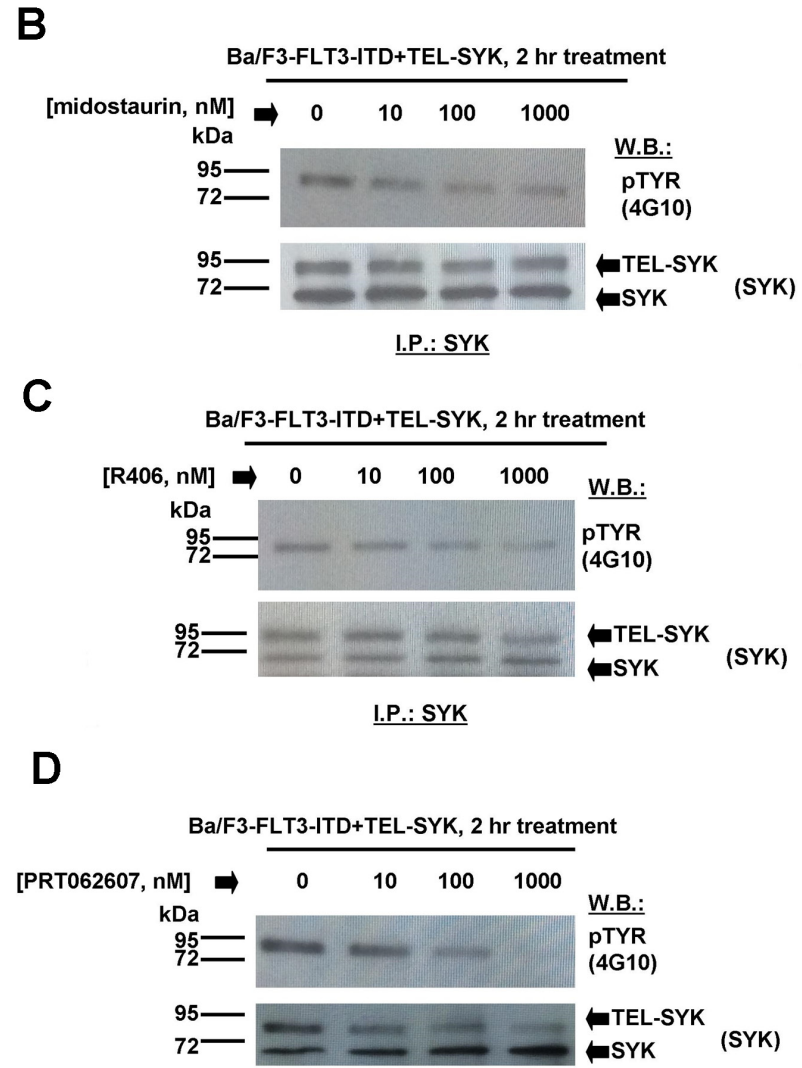

(SYK)

I.P.: SYK

Figure 4: Effects of midostaurin, R406, and PRT062607 on phosphorylation of constitutively activated TEL-SYK and downstream signaling molecules. A. Effect of midostaurin and PRT062607 on TEL-SYK phosphorylation and phosphorylation of downstream effectors following $2 \mathrm{hr}$ of treatment. For the experiment analyzing effects of midostaurin and PRT062607 on phosphorylation of TEL-SYK, SYK immunoprecipitation was carried out prior to immunoblotting with a PTYR (4G10) antibody. Immunoblotting was performed for the experiment analyzing effects of midostaurin and PRT062607 on signaling molecules downstream of TEL-SYK. Relative densitometry readings for $\mathrm{pMAPK} /$ total MAPK (normalized to control lane) are as follows: $0 \mathrm{nM}$ midostaurin=1.0, $10 \mathrm{nM}$ midostaurin=0.93, $100 \mathrm{nM}$ midostaurin=0.41, $1000 \mathrm{nM}$ midostaurin=0.04, $0 \mathrm{nM}$ PRT062607=1.0, $10 \mathrm{nM}$ PRT062607=0.72, $100 \mathrm{nM}$ PRT062607=0.37, 1000 nM PRT062607=0.07. B.-D. Effects of midostaurin, R406, and PRT062607 on TEL-SYK phosphorylation in Ba/F3 cells co-expressing FLT3-ITD and TEL-SYK. For these experiments, SYK immunoprecipitation was carried out prior to immunoblotting with a PTYR (4G10) antibody. ImageJ 32 software was used for densitometry. Briefly, to get band intensities, the area of all the bands was first measured, and then normalized to the control lane, and then normalized to total protein. 
Table 2: Combination indices generated by Calcusyn software for midostaurin+/- R406, R788, and PRT062607 tested against Ba/F3-SYK-TEL, Ba/F3-TEL-SYK, Ba/F3-FLT3-ITD, Ba/F3-FLT3-ITD+SYK-TEL, Ba/F3-FLT3-ITD+TELSYK, MOLM14, MOLM13- luc+, MV4,11, and midostaurin-resistant MOLM13-luc+ cells.

\begin{tabular}{|c|c|c|c|c|}
\hline Cell Line, Drug Treatments & ED25 & ED50 & ED75 & ED90 \\
\hline \multicolumn{5}{|l|}{ Ba/F3-SYK-TEL } \\
\hline midostaurin+R406 & 0.39943 & 0.51171 & 0.65589 & 0.84114 \\
\hline midostaurin+R788 & 0.52255 & 0.65469 & 0.82026 & 1.02774 \\
\hline midostaurin+PRT062607 & 0.80590 & 0.87952 & 0.96109 & 1.05159 \\
\hline \multicolumn{5}{|l|}{ Ba/F3-TEL-SYK } \\
\hline midostaurin+R406 & 0.76376 & 0.85078 & 0.94864 & 1.05881 \\
\hline midostaurin+R788 & 0.73959 & 0.82537 & 0.92131 & 1.0286 \\
\hline midostaurin+PRT062607 & 0.13909 & 0.21598 & 0.33684 & 0.52773 \\
\hline \multicolumn{5}{|l|}{ Ba/F3-FLT3-ITD } \\
\hline midostaurin+R406 & 0.74152 & 0.74592 & 0.75824 & 0.77845 \\
\hline midostaurin+R788 & 0.47348 & 0.53233 & 0.60096 & 0.68133 \\
\hline midostaurin+PRT062607 & 0.54961 & 0.54046 & 0.53808 & 0.54124 \\
\hline \multicolumn{5}{|l|}{ Ba/F3-FLT3-ITD+SYK-TEL } \\
\hline midostaurin+R406 & 0.31570 & 0.43963 & 0.61232 & 0.85301 \\
\hline midostaurin+R788 & 0.85128 & 0.99349 & 1.15950 & 1.35328 \\
\hline midostaurin+PRT062607 & 0.86375 & 0.97146 & 1.09260 & 1.22885 \\
\hline \multicolumn{5}{|l|}{ Ba/F3-FLT3-ITD+TEL-SYK } \\
\hline midostaurin+R406 & 0.73305 & 0.82401 & 0.92631 & 1.04135 \\
\hline midostaurin+R788 & 0.77081 & 0.84045 & 0.91657 & 0.99981 \\
\hline midostaurin+PRT062607 & 0.48694 & 0.60149 & 0.74306 & 0.91802 \\
\hline \multicolumn{5}{|l|}{ MOLM14 } \\
\hline midostaurin+R406 & 0.42615 & 0.54750 & 0.71916 & 0.96943 \\
\hline midostaurin+R788 & 0.55385 & 0.66601 & 0.82207 & 1.04052 \\
\hline midostaurin+PRT062607 & 0.88142 & 0.92061 & 0.96929 & 1.02933 \\
\hline \multicolumn{5}{|l|}{ MOLM13-luc+ } \\
\hline midostaurin+R406 & 0.28109 & 0.39832 & 0.56512 & 0.80281 \\
\hline midostaurin $+\mathrm{R} 788$ & 0.46901 & 0.55688 & 0.66214 & 0.78838 \\
\hline midostaurin+PRT062607 & 0.75206 & 0.84920 & 0.96037 & 1.08776 \\
\hline \multicolumn{5}{|l|}{ MV411 } \\
\hline midostaurin $+\mathrm{R} 406$ & 0.85296 & 0.85000 & 0.84890 & 0.84963 \\
\hline midostaurin+R788 & 0.74899 & 0.78910 & 0.87103 & 1.00545 \\
\hline midostaurin+PRT062607 & 1.28030 & 1.18799 & 1.10918 & 1.04150 \\
\hline \multicolumn{5}{|l|}{ midostaurin-resistant MOLM13-luc+ } \\
\hline midostaurin+R406 & 0.94762 & 1.00537 & 1.06943 & 1.14050 \\
\hline midostaurin+R788 & 1.16299 & 1.09052 & 1.02404 & 0.96302 \\
\hline midostaurin+PRT062607 & 0.46223 & 0.59674 & 0.77041 & 0.99465 \\
\hline \multicolumn{5}{|l|}{ Ba/F3-FLT3-ITD } \\
\hline crenolanib+PRT062607 & 1.06052 & 1.09187 & 1.12443 & 1.15826 \\
\hline \multicolumn{5}{|l|}{ Ba/F3-FLT3-ITD+SYK-TEL } \\
\hline crenolanib+PRT062607 & 0.93074 & 0.93282 & 0.94592 & 0.97158 \\
\hline
\end{tabular}

Also shown are combination indices generated by Calcusyn software for crenolanib+/-PRT062607 tested agianst Ba/F3FLT3-ITD and Ba/F3-FLT3-ITD+SYK-TEL. Proliferation assays/combination studies were carried out for approximately 3 days 
6D-6E, 7D-7E, 8D-8E). Combination effects of these drug combinations also correlated with increased caspase-3 and -7 activity, suggesting increased apoptosis in drug combination-treated cells (Figures 6-8F).

The combination of midostaurin+/-R406, midostaurin+/-R788, and midostaurin+/-PRT062607 also led to increased inhibition of the growth of Ba/F3-FLT3ITD cells (Supplementary Figure 6A-C). Importantly, combination effects observed were confirmed not to be unique to the $\mathrm{Ba} / \mathrm{F} 3$ system. Positive combination effects were also observed between midostaurin +/- R406, R788, or PRT062607 against human FLT3-ITD-driven MOLM13, MOLM14, and MV411 cells (Figure 9A-9F, Supplementary Figure 6D-6F and Table 2). Increases in G1 arrest and/or percent of apoptotic cells and fraction of cells in subG0/G1 were observed for FLT3-ITD-driven cells treated with combinations of midostaurin and R406 or R788 (Figure 9G-9J and Supplementary Figure 7). It should be noted that, in contrast to drug combinations against other cell lines, generally no additive to synergistic effects were observed for midostaurin and PRT062607 against the MV4,11 cell line (Supplementary Figure 6F).

We sought to confirm that potentiation of midostaurin by dual FLT3/SYK inhibition and targeted SYK inhibition could be advantageous in terms of overriding some forms of drug resistance. Midostaurinresistant MOLM13-luc+ cells were previously established via prolonged exposure to midostaurin, and exhibited increased levels of cell surface FLT3 protein and elevated levels of phospho-MAPK, however normal levels of phospho-STAT5 [28]. Midostaurin-resistant MOLM13luc + cells were tested with combinations of midostaurin and R406, R788, or PRT062607, and nearly additive effects were observed between midostaurin $+/-$ R406 or R788 against the drug-resistant cells across a range of concentrations (Figure 10A-10B and Table 2). Positive combination effects between midostaurin and PRT062607 against these cells were more striking, with combination indices indicative of synergy (Figure 10C and Table 2). Midostaurin-resistant MOLM13-luc+ cells are less responsive to midostaurin than parental MOLM13-luc+ as evidenced by a rightward shift in the midostaurin doseresponse curve (Figure 10D). Drug resistance is likely due to the over-expression of FLT3 protein in the midostaurinresistant cells, as previously reported and also confirmed in the present study (Figure 10E). SYK protein expression,
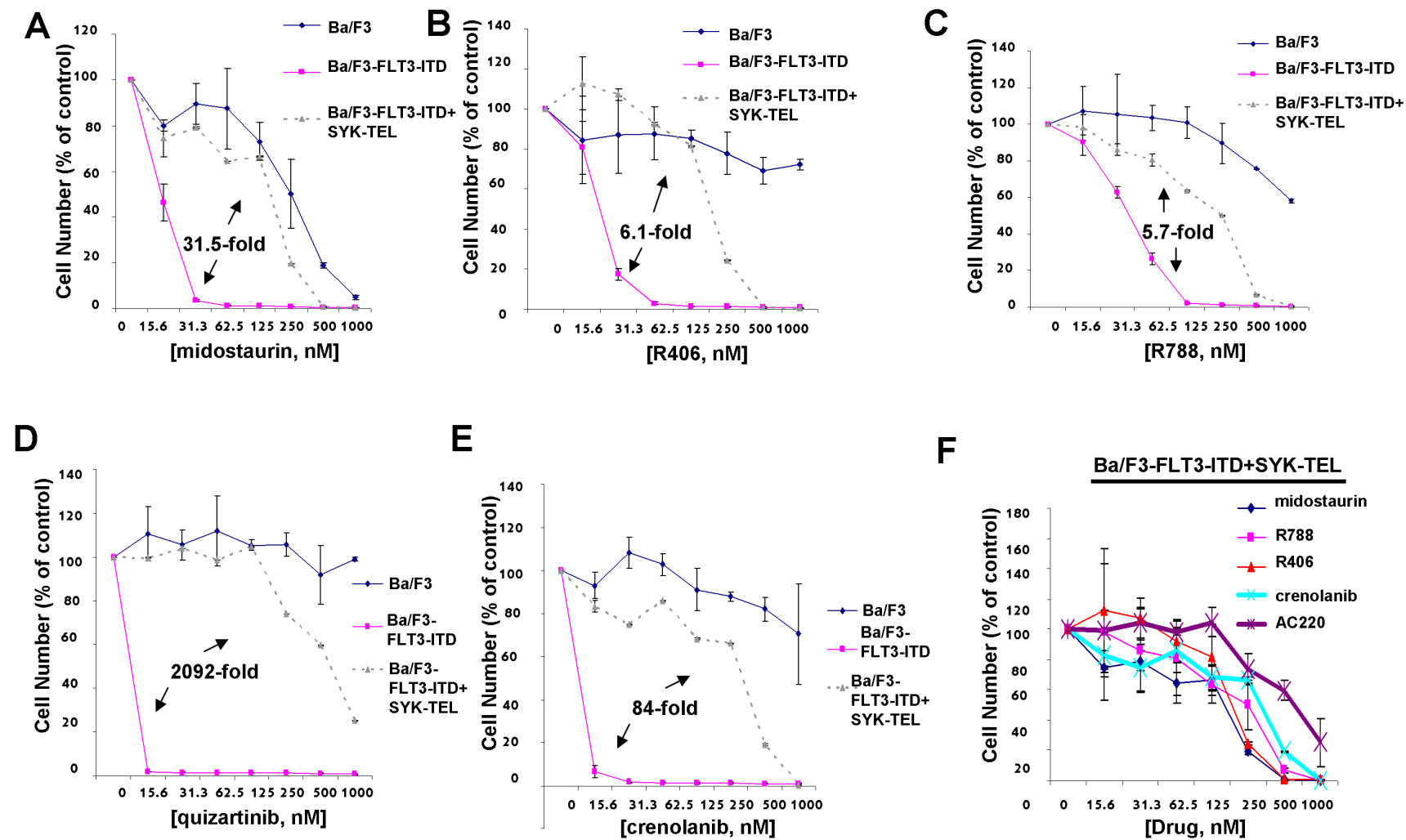

Figure 5: Comparison of effects of targeted FLT3 inhibitors with dual SYK/FLT3 inhibitors on cells expressing FLT3-ITD and activated SYK. A.-E. Three-day treatment of Ba/F3, Ba/F3-FLT3-ITD, and Ba/F3-FLT3-ITD+SYK-TEL cells with midostaurin (A), R406 (B), R788 (C), quizartinib (D), or crenolanib (E). F. Comparison of effects of dual SYK/FLT3 inhibitors versus selective FLT3 inhibitors on growth of Ba/F3 cells co-expressing highly activated SYK-TEL and FLT3-ITD following 3 days of treatment. Error bars represent the mean+/-S.D. for all proliferation studies. Fold differences between IC50s for inhibitor treatment of Ba/F3-FLT3ITD and Ba/F3-FLT3-ITD+SYK-TEL are shown for all of the graphs. Parental Ba/F3 cells are shown as a control for nonspecific toxicity. 
however, remained the same between the two lines (Figure $10 \mathrm{E})$.

We next evaluated the therapeutic potential of combining midostaurin with dual SYK/FLT3 or SYK inhibitors by testing the resulting growth inhibitory effect on FLT3-ITD-expressing AML primagraft cells from relapsed patients or patients that were refractory to chemotherapy treatment. As normal controls, PBMC cells from a healthy donor were tested with the combination of midostaurin+R406, and little to no inhibitory effects of the agents alone or combined were observed (Figure 11A). MOLM14 cells were tested in parallel with the normal PBMC cells as a positive control for drug activity (Figure 11B). We then tested the ability of R406 to potentiate midostaurin (Figure 11C). Whereas little to no single agent activity was observed for midostaurin at up to 20
$\mathrm{nM}$ or R406 at up to $100 \mathrm{nM}$ against AML primagraft sample \#1 (disease stage at time of sample acquisition: relapsed post-allogeneic HSCT, Supplementary Table 1), the combination of midostaurin and R406 led to a higher percentage of cell death as compared to either single agent across the full range of concentrations (Figure 11C). A second AML primagraft sample (\#2) (disease stage at time of sample acquisition: relapsed after multiple modalities, Supplementary Table 2) was tested with midostaurin+/-PRT062607 at concentrations previously tested against FLT3-ITD-expressing cell lines (Figure 11D). Whereas little to no single agent activity was observed for midostaurin at up to $40 \mathrm{nM}$, PRT062607 treatment led to around $50 \%$ cell death at $1000 \mathrm{nM}$, and a slight leftward shift in the dose-response curve (indicating a positive drug combination effect) was observed for the

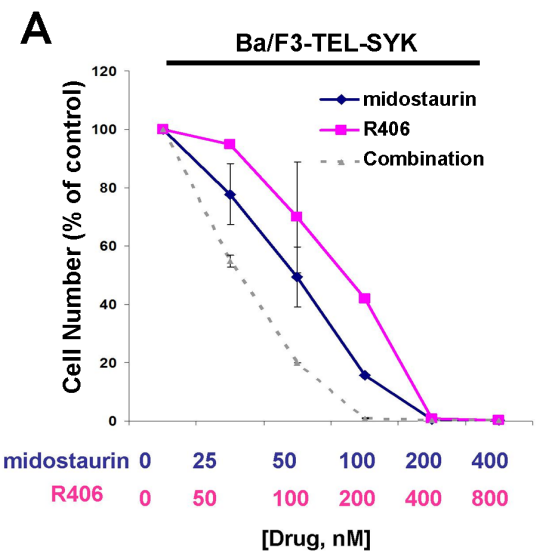

D

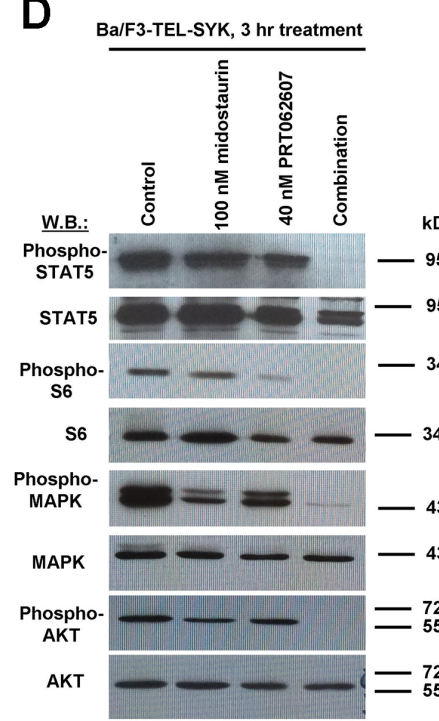

B

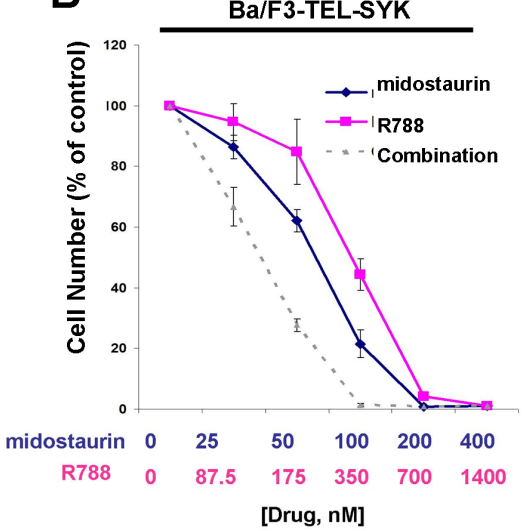

E

Ba/F3-TEL-SYK, 3 hr treatment

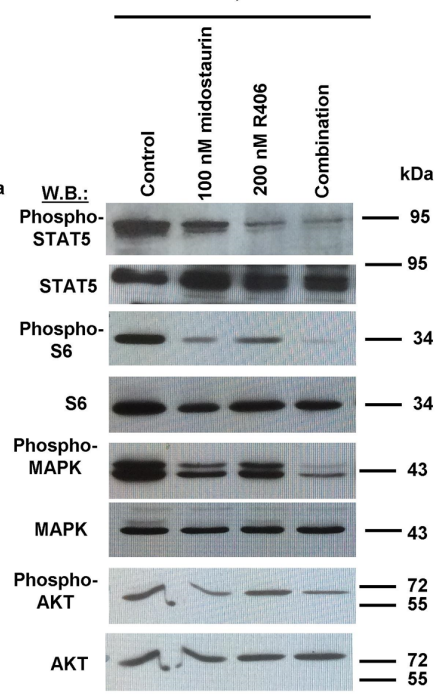

C
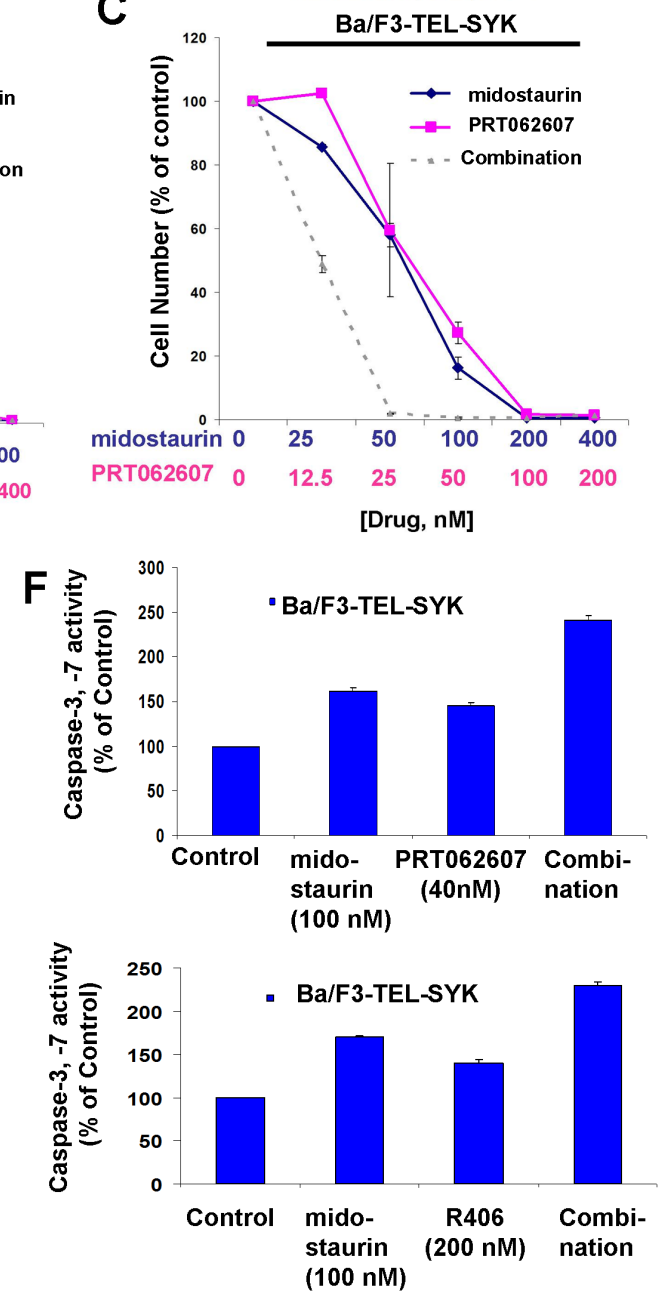

Figure 6: Potentiation of effects of midostaurin against Ba/F3-TEL-SYK cells by R406, R788, and PRT062607. A.C. Three-day treatments of Ba/F3-TEL-SYK cells with midostaurin+/-R406, midostaurin+/-R788, or midostaurin+/-PRT062607. Error bars represent the mean+/-S.D. for all proliferation studies. D. Effects of midostaurin (100 nM), PRT062607 (40 nM), or a combination on signaling molecules downstream of TEL-SYK. E. Effects of midostaurin (100 nM), R406 (200 nM), or a combination on signaling molecules downstream of TEL-SYK. Immunoblotting was performed for the experiment analyzing effects of midostaurin+/-PRT062607 and midostaurin+/-R406 on signaling molecules downstream of TEL-SYK. F. Caspase activity in Ba/F3-TEL-SYK cells treated with midostaurin +/- PRT062607 or midostaurin +/- R406. 
drug combination (Figure 11D). Interestingly, treatment of a third AML primagraft sample (\#3) (disease stage at time of sample acquisition: primary refractory postinduction, Supplementary Table 3 ) with midostaurin (up to $40 \mathrm{nM}$ ), alone and in combination with PRT062607 across a comparatively conservative concentration range (up to only $400 \mathrm{nM}$, a 10-fold lower concentration than was used against FLT3-ITD-positive cell lines), resulted in a higher percentage in the combination of agents as compared to either agent alone (Figure 11E). SYK protein expression was detectable in all FLT3-ITD-positive AML primagrafts tested; SYK expression in bone marrow and spleen samples corresponding to the primagrafts is shown alongside normal PBMC cells (Figure 11F).
Potentiation of the effects of crenolanib by targeted SYK suppression against cells expressing FLT3-ITD and activated SYK

Given that dual SYK/FLT3 inhibition and targeted SYK inhibition potentiated the effects of midostaurin, an established dual inhibitor of SYK and FLT3, against cells driven by FLT3-ITD, activated SYK, or a combination of both, we were interested in investigating the ability of additional SYK inhibition to similarly augment the effectiveness of targeted FLT3 inhibition. The combination of crenolanib with PRT062607 was nearly additive against $\mathrm{Ba} / \mathrm{F} 3-\mathrm{FLT} 3-\mathrm{ITD}$ cells for the concentrations of inhibitors at which $25 \%$ and $50 \%$ of cell growth inhibition is observed (ED25-ED50) (Figure 12A and Table 2). PRT062607 potentiated the anti-leukemic
A

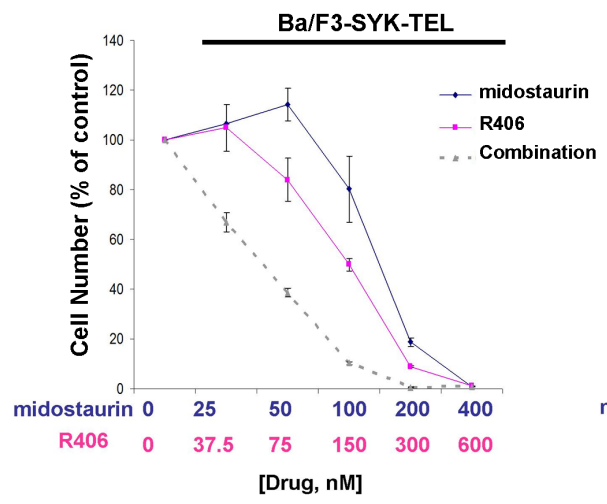

B

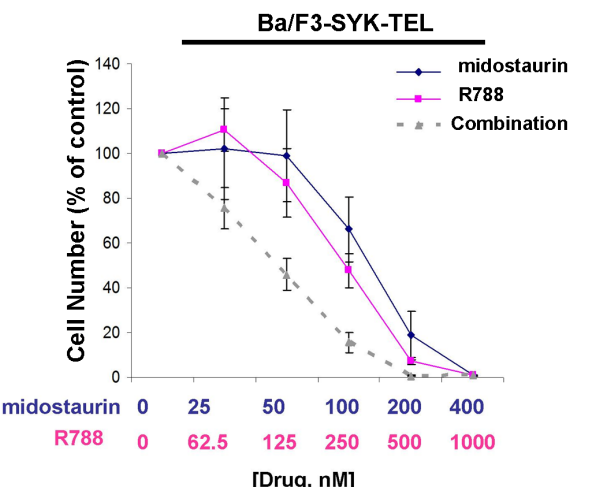

C

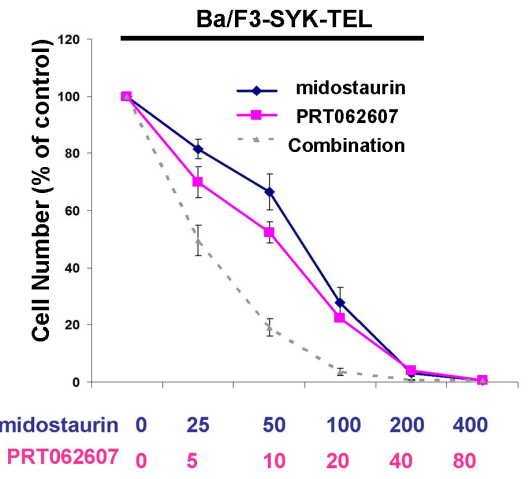

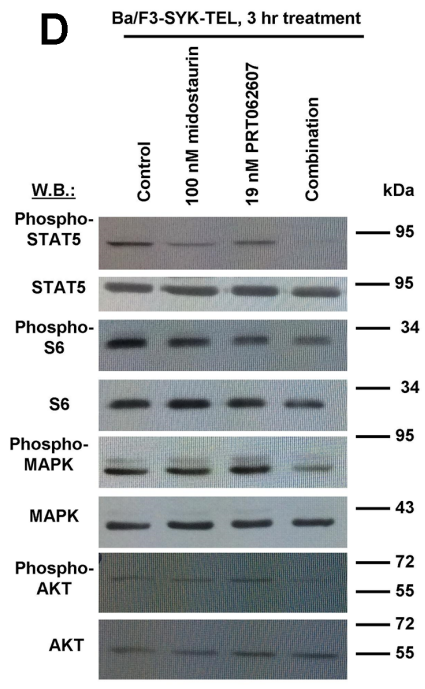
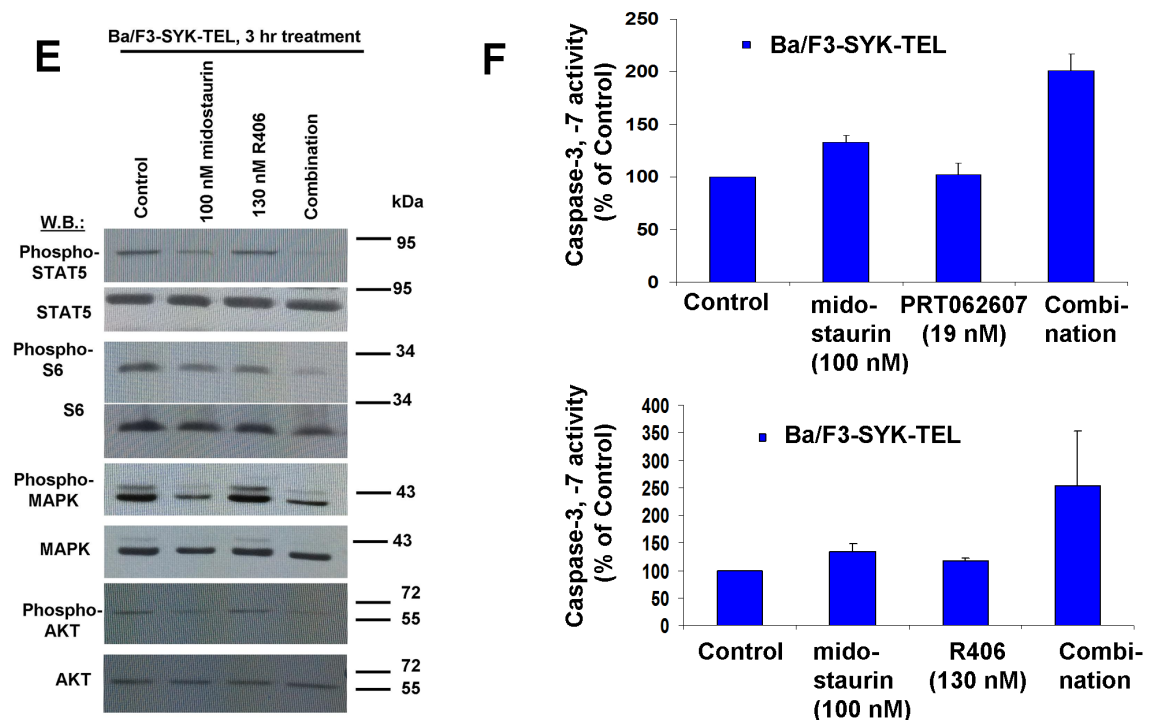

Figure 7: Potentiation of effects of midostaurin against Ba/F3-SYK-TEL cells by R406, R788, and PRT062607. A.C. Three-day treatments of Ba/F3-SYK-TEL cells with midostaurin+/-R406, midostaurin+/-R788, or midostaurin+/-PRT062607. D. Effects of midostaurin $(100 \mathrm{nM})$, PRT062607 (19 nM), or a combination on signaling molecules downstream of SYK-TEL. Error bars represent the mean+/-S.D. for all proliferation studies. E. Effects of midostaurin (100 nM), R406 (130 nM), or a combination on signaling molecules downstream of SYK-TEL. Immunoblotting was performed for the experiment analyzing effects of midostaurin+/-PRT062607 and midostaurint/-R406 on signaling molecules downstream of SYK-TEL. F. Caspase activity in Ba/F3-SYK-TEL cells treated with midostaurin +/- PRT062607 or midostaurin +/- R406. 
effects of crenolanib against cells co-expressing FLT3ITD+SYK-TEL, with nearly additive effects observed for concentrations of inhibitors at which $25 \%, 50 \%, 75 \%$, and $90 \%$ of cell growth inhibition is observed (ED25-ED90) (Figure 12B and Table 2).

\section{DISCUSSION}

SYK has been shown to be important in FLT3 mutant-positive AML in that it transactivates FLT3-ITD, and through over-expression has been shown to play a role in AML transformation and resistance to FLT3 inhibition [10]. In addition, the combined inhibition of FLT3 by a targeted FLT3 inhibitor (quizartinib) and SYK has been demonstrated to be more effective than FLT3 inhibition alone [10]. Midostaurin and its metabolites, CGP52421 and CGP62221, have been reported as inhibitors of SYK in advanced systemic mastocytosis [25]. However, the activity of midostaurin as an inhibitor of SYK has not yet been explored in FLT3-mutant-positive AML.

Midostaurin, which has recently been FDA approved, was shown in a large trial (RATIFY (CALGB 10603)) in newly diagnosed patients to increase survival when combined with standard chemotherapy [7]. This study supports the notion that inhibition of FLT3 is important in patients with mutations in the FLT3 gene, and also highlights the clinical effectiveness of midostaurin as a therapeutic for AML. As highly activated SYK has been found to occur at a higher frequency in AML patients harboring the ITD mutant than patients expressing wt
A

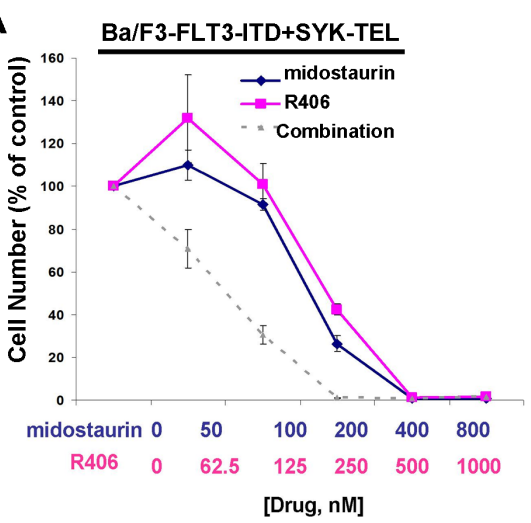

D

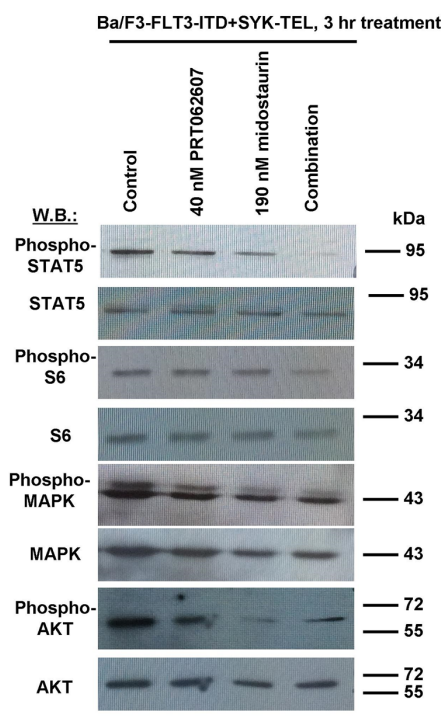

B

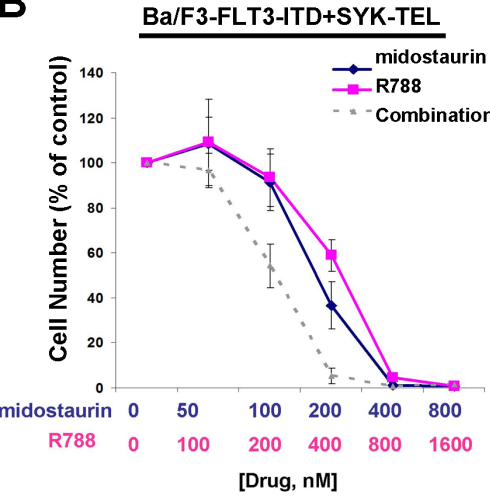

C

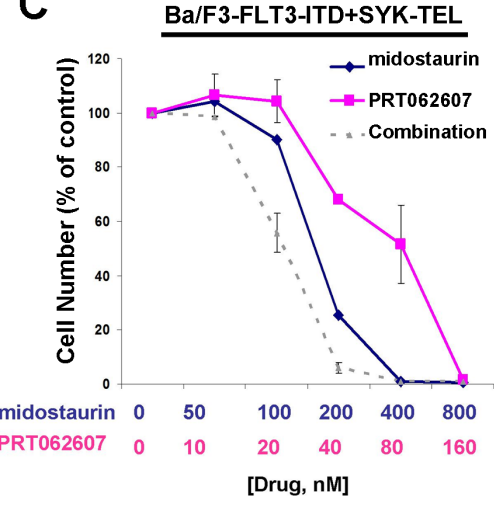

E

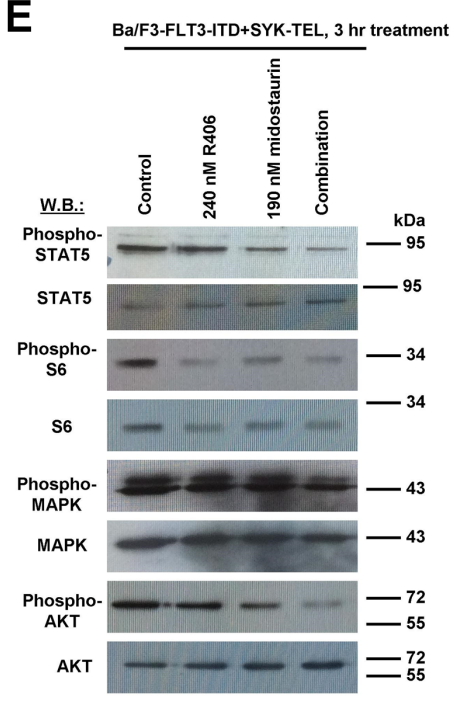

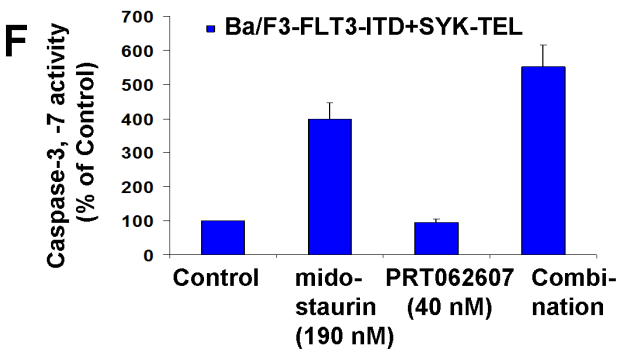

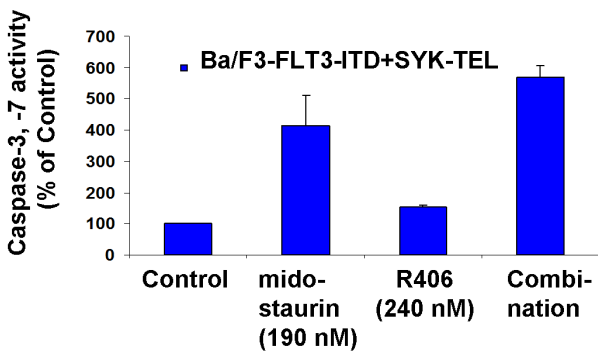

Figure 8: Potentiation of effects of midostaurin against Ba/F3-FLT3-ITD+SYK-TEL cells by R406, R788, and PRT062607. A.-C. Three-day treatments of Ba/F3-FLT3-ITD+SYK-TEL cells with midostaurin+/-R406, midostaurin+/-R788, or midostaurin+/-PRT062607. D. Effects of midostaurin (190 nM), PRT062607 (40nM), or a combination on signaling molecules downstream of Ba/F3-FLT3-ITD+SYK-TEL. Error bars represent the mean+/-S.D. for all proliferation studies. E. Effects of midostaurin (190 nM), R406 (240 nM), or a combination on signaling molecules downstream of Ba/F3-FLT3-ITD+SYK-TEL. Immunoblotting was performed for the experiment analyzing effects of midostaurin+/-PRT062607 and midostaurin+/-R406 on signaling molecules downstream of FLT3ITD+SYK-TEL. F. Caspase activity in Ba/F3-FLT3-ITD+SYK-TEL cells treated with midostaurin +/- PRT062607 or midostaurin +/R406. 
FLT3 [10], we were interested in exploring the activity of midostaurin against SYK using cell-based models of SYK-driven and SYK- and FLT3-driven leukemia. We found that while midostaurin is an excellent inhibitor of FLT3-ITD-expressing cells, it is a relatively weak inhibitor of activated SYK alone (SYK-TEL or TEL-SYK) and activated SYK (SYK-TEL) co-expressed with FLT3ITD. Specifically, we found the potency of midostaurin against activated SYK-expressing cells to be 2.5 to 5 -fold less than that of the targeted SYK inhibitor, PRT062607. Conversely, the potency of midostaurin against cells expressing FLT3-ITD or FLT3-ITD+TEL-SYK (which are believed to be driven predominantly by FLT3-ITD due to a lack of transactivation of FLT3 by TEL-SYK) was close to 100-fold higher than PRT062607. Thus, the benefit of dual suppression of FLT3 and SYK by midostaurin allows this agent to be more versatile than a targeted SYK inhibitor in that it is effective against cells expressing oncogenic FLT3, activated SYK, or cells expressing both oncoproteins.

Having established the extent by which SYK is inhibited by midostaurin in addition to FLT3 in comparison to the dual SYK/FLT3 inhibitors, R406 and R788, we were then interested in exploring whether or not additional SYK inhibition is able to further improve the efficacy of midostaurin. Potentiation of the effects of midostaurin by R406 or PRT062607, as measured in cell growth assays and evidenced by increased killing of constitutively activated SYK and/or mutant FLT3expressing cells, correlated with increased suppression of signaling molecules, including those involved in the phosphatidylinositol 3-kinase pathway and mitogenactivated protein kinase signaling, characterized as phosphorylated substrates and exerting effects downstream of either oncogenic FLT3 or activated SYK [29, 30, 5, 24].
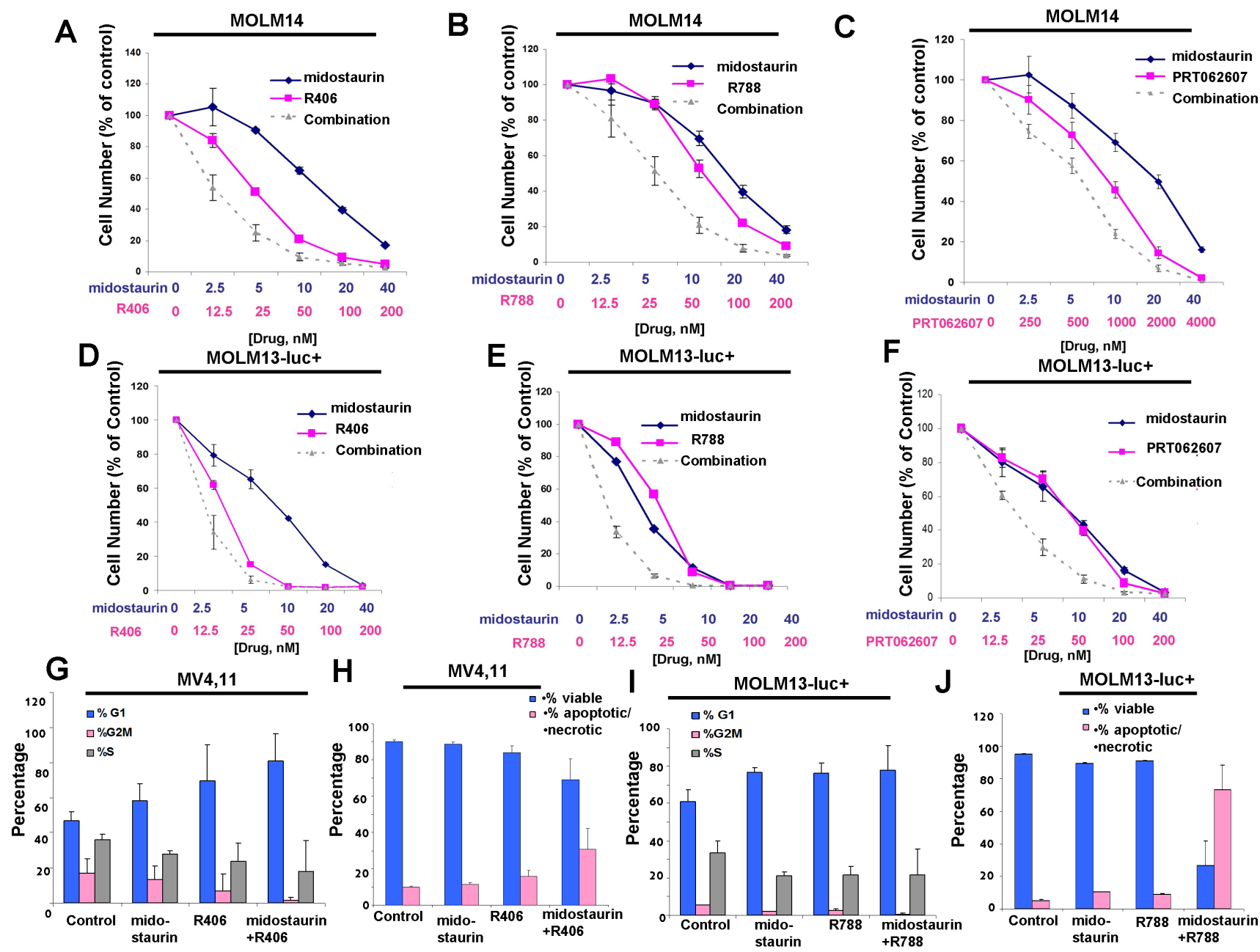

Figure 9: Potentiation of midostaurin by R406, R788 and PRT062607 against FLT3-ITD-driven cell lines. A.-C. Three-day treatments of MOLM14 cells with midostaurin+/-R406, midostaurin+/-R788, or midostaurin+/-PRT062607. D.-F. Three-day treatments of MOLM13-luc+ cells with midostaurin+/-R406, midostaurin+/-R788, or midostaurin+/-PRT062607. Error bars represent the mean+/-S.D. for all proliferation studies. G.-H. Effects of midostaurin (10 nM) +/-R406 (50 nM) on MV4,11 cell cycle progression (G) and apoptosis (H). I.-J. Effects of midostaurin (10 nM) +/- R788 (50 nM) on MOLM13-luc+ cell cycle progression (I) and apoptosis (J). Error bars represent the mean+/-S.D. 

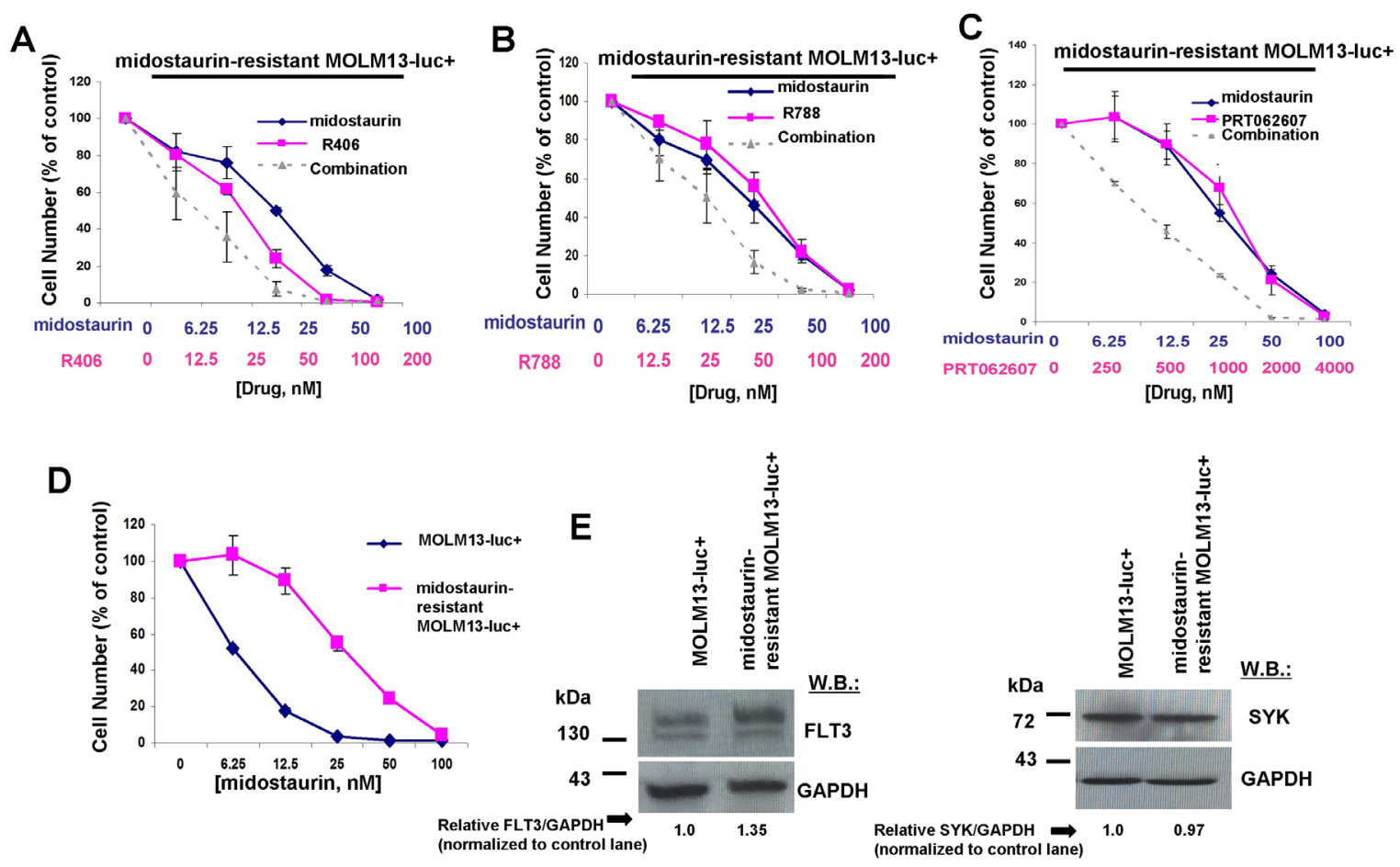

Figure 10: Potentiation of effects of midostaurin against FLT3 inhibitor-resistant cells by R406, R788, and PRT062607. A.-C. Three-day treatments of midostaurin-resistant MOLM13-luc+ cells with midostaurin+/-R406, midostaurin+/-R788, or midostaurin+/PRT062607. D. Three-day treatment of MOLM13-luc+ and midostaurin-resistant MOLM13-luc+ cells with midostaurin. Error bars represent the mean+/-S.D. for all proliferation studies. E. Comparison of FLT3 and SYK expression levels, determined by immunoblotting, in MOLM13 versus midostaurin-resistant MOLM13 cells. ImageJ 32 software was used for densitometry. Briefly, to get band intensities, the area of all the bands was first measured, and then normalized to the control lane, and then normalized to total protein.
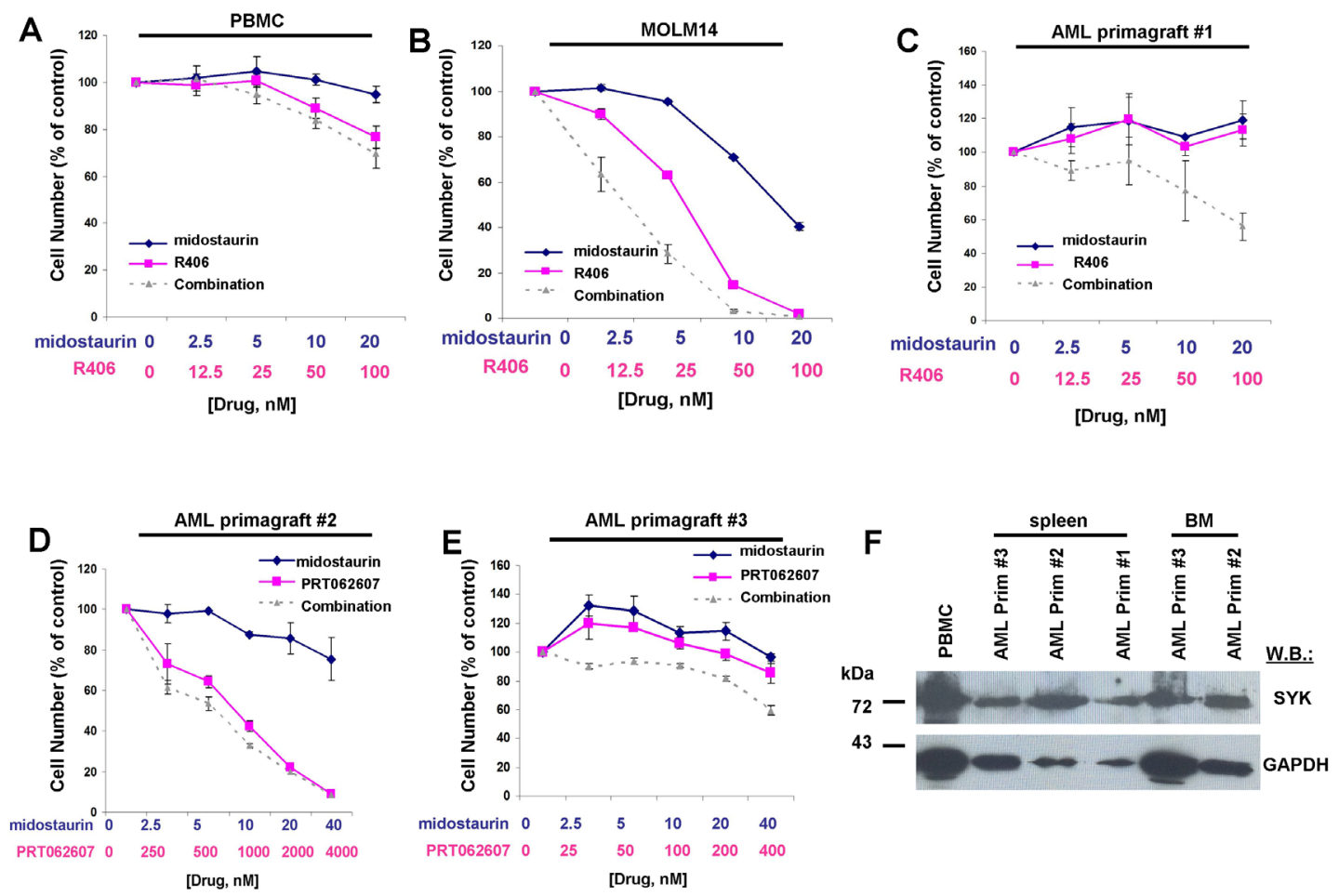

Figure 11: Potentiation of effects of midostaurin against drug-resistant AML primagraft cells by R406 and PRT062607. A.-B. Three-day treatment of normal PBMCs (A) with midostaurin+/-R406. MOLM14 cells (B) were treated in parallel as a positive control for drug effectiveness. C.-E. Three-day treatment of mutant FLT3-positive AML primagrafts with midostaurin+/-R406 (C) and midostaurin+/-PRT062607 (D-E). F. SYK protein expression in normal PBMC cells and FLT3-ITD-positive AML primagraft cells. 
Our results are consistent with earlier findings showing that, in a SYK-TEL cooperative model with FLT3-ITD, the combination of PRT062607 and quizartinib increased survival of mice, which correlated with inhibition of FLT3-ITD and SYK activation [10]. Importantly, our results also suggest that the effects of midostaurin can be potentiated by dual FLT3/SYK suppression or more targeted SYK suppression in the context of midostaurinresistant leukemia and in FLT3-ITD-expressing primary cells.

Drug combination effects were observed against phosphorylated STAT5, S6, MAPK, and AKT by midostaurin+R406 or midostaurin+PRT062607 in cells expressing constitutively activated SYK. Significantly, STAT5 has been identified as a major downstream signaling mediator of constitutively activated SYK, both in vitro and in vivo, and targeted deletion of Stat5 in vivo was shown to fully abrogate AML caused by TEL-SYK [24]. These results support the notion of STAT5 as a key driver of SYK-induced cellular transformation, and shed light on the potential clinical importance of our observation of enhanced suppression of activated STAT5 by midostaurin combined with inhibitors of FLT3 and SYK or SYK alone.

Importantly, the efficacy of midostaurin, R406 and R788 toward cells expressing FLT3-ITD alone or FLT3-ITD with activated SYK was compared with the efficacy of targeted FLT3 inhibitors quizartinib and crenolanib, neither of which targets SYK. We observed that all of the inhibitors were highly potent toward FLT3-ITD-driven cells in the absence of activated SYK, however midostaurin, R406 and R788 showed relatively higher potency toward cells expressing activated SYK.

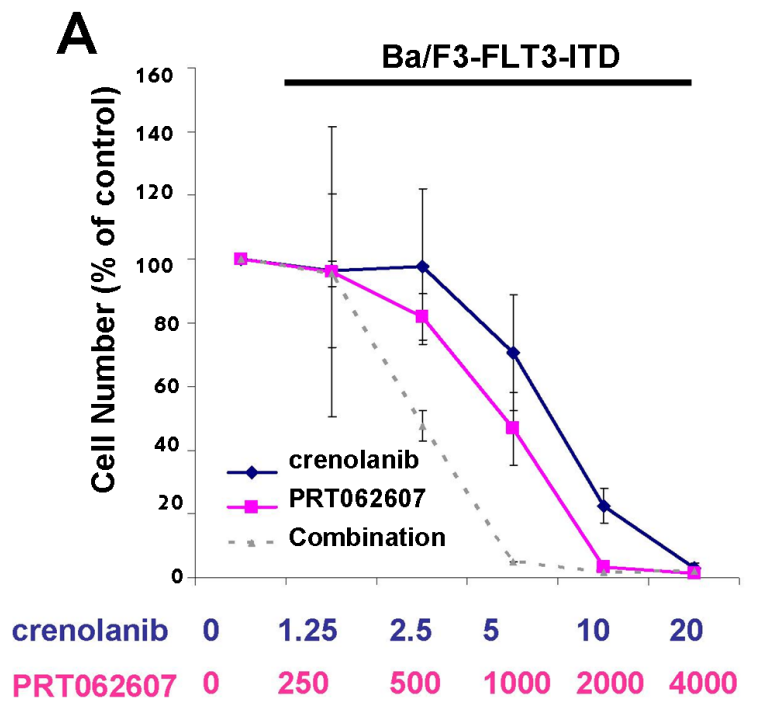

[Drug, nM]
Whereas inhibition of SYK alone does not substitute for FLT3 inhibition and is incapable of inducing death of cells predominantly driven by FLT3-ITD, additional SYK inhibition was observed to potentiate the antiproliferative activity of both multi-targeted and selective FLT3 inhibitors against cells driven by activated SYK. Taken together, the ability of SYK and dual FLT3/SYK suppression to enhance the growth inhibitory effects of midostaurin and other FLT3 inhibitors in the context of both kinase inhibitor-sensitive and kinase inhibitorresistant disease warrants further investigation for the continued development and optimization of midostaurin as a treatment strategy for AML.

\section{MATERIALS AND METHODS}

\section{Chemical compounds and biologic reagents}

Midostaurin was synthesized by Novartis Pharma AG, Basel, Switzerland. R406, R788, PRT062607, crenolanib and quizartinib were purchased from Selleckchem (Houston, TX) and dissolved in DMSO to obtain a $10 \mathrm{mM}$ stock solution. Serial dilutions were then made, to obtain final dilutions for cellular assays with a final concentration of DMSO not exceeding $0.1 \%$.

\section{Cell lines and cell culture}

$\mathrm{Ba} / \mathrm{F} 3$ (interleukin [IL]-3-dependent murine pro-B) cells engineered to express FLT3-ITD, SYK-TEL, TEL-

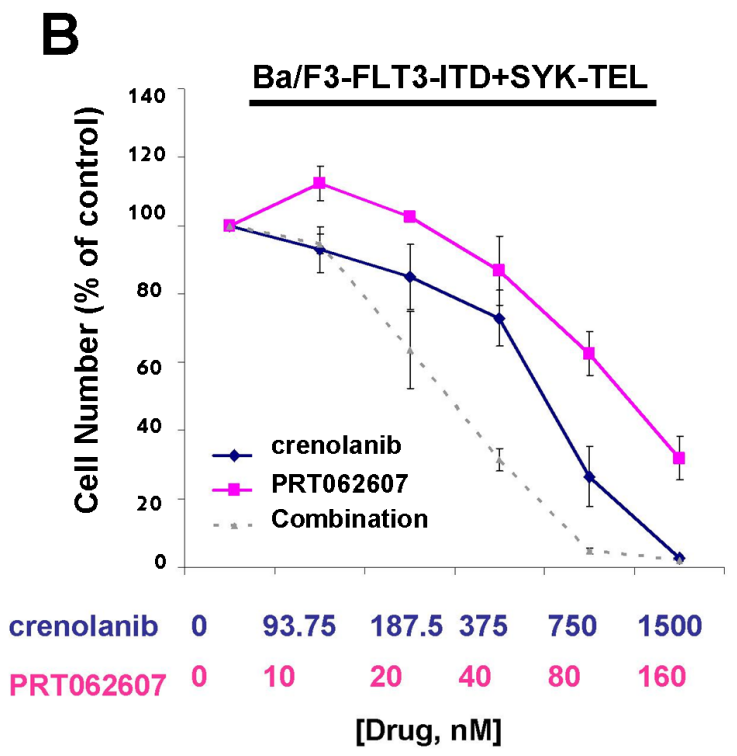

Figure 12: Potentiation of effects of crenolanib by PRT062607 against Ba/F3-FLT3-ITD and Ba/F3-FLT3-ITD+SYKTEL cells. A.-B. Three-day treatment of Ba/F3-FLT3-ITD (A) or Ba/F3-FLT3-ITD+SYK-TEL (B) cells with crenolanib+/-PRT062607. Error bars represent the mean+/-S.D. for all proliferation studies. 
SYK, FLT3-ITD+SYK-TEL, and FLT3-ITD+TEL-SYK [10] were provided by Dr. Kimberly Stegmaier. Ba/ F3 cells were engineered to over-express wt FLT3 as previously described [6].

Human AML-derived, FLT3-ITD-expressing MV4,11 cells were obtained from Dr. Anthony Letai. The human AML-derived, FLT3-ITD-expressing line, MOLM14 [31], was provided to us by Dr. Scott Armstrong, Dana-Farber Cancer Institute (DFCI), Boston, MA.

The human AML-derived, FLT3-ITD-expressing cell line, MOLM-13 (DSMZ (German Resource Centre for Biological Material), was engineered to express luciferase fused to neomycin phosphotransferase (pMMP-LucNeo) by transduction with a VSVG-pseudotyped retrovirus as previously described [32]. Development of PKC412resistant cells derived from long-term culture or drugresistant colonies (MOLM13-R-PKC412 (CFU)) was described previously [28].

All cell lines used in this study were cultured with $5 \% \mathrm{CO}_{2}$ at $37^{\circ} \mathrm{C}$, at a concentration of $2 \times 10^{5}$ to $5 \times 10^{5}$ in RPMI (Mediatech, Inc., Herndon, VA) with $10 \%$ fetal bovine serum (FBS) and supplemented with $2 \%$ L-glutamine and $1 \%$ penicillin/streptomycin. Parental Ba/ F3 cells and Ba/F3-wt FLT3 cells were cultured in RPMI with $10 \%$ FBS and supplemented with $2 \%$ L-glutamine and $1 \%$ penicillin/streptomcyin, as well as 20\% WEHI (as a source of IL-3).

Human cell lines were submitted for cell line authentication and were authenticated within 6 months of manuscript preparation through cell line short tandem repeat (STR) profiling (DDC Medical, Fairfield, $\mathrm{OH}$ and Molecular Diagnostics Laboratory, Dana-Farber Cancer Institute). All cell lines tested matched $>80 \%$ with lines listed in the ATCC or DSMZ Cell Line Bank STR. All cell lines were confirmed to be virus- and Mycoplasma-free.

\section{Immunoblotting and immunoprecipitation}

Protein lysate preparation, immunoblotting, and immunoprecipitation were carried out as previously described [6]. Briefly, to obtain protein lysates for immunoprecipitation and immunoblotting, cells were lysed in lysis buffer $(0.02 \mathrm{M}$ Tris $[\mathrm{pH} 8.0], 0.15 \mathrm{M}$ $\mathrm{NaCl}, 10 \%$ glycerol, $1 \% \mathrm{NP}-40$ (wt/vol), $0.1 \mathrm{M} \mathrm{NaF}, 1$ $\mathrm{mM}$ phenylmethylsulfonyl fluoride ( $\mathrm{PhCH} 2 \mathrm{SO} 2), 1 \mathrm{mM}$ sodium orthovanadate (Na3VO4), and HALT $^{\mathrm{TM}}$ protease inhibitor cocktail, EDTA-free (100X) (ThermoFisher Scientific, Waltham, MA). Protein lysates were incubated on ice for $25 \mathrm{~min}$, vortexed at $5 \mathrm{~min}$ intervals, and then centrifuged for $15 \mathrm{~min}$ at $12,000 \mathrm{X} \mathrm{g}$. Supernatants were saved, and the Bio-Rad Protein Assay was used to determine protein yields (Bio-Rad Laboratories, Hercules, CA). Equivalent amounts of protein were then loaded onto a gel for immunoblotting. For immunoprecipitation, cell lysates were incubated with antibody and protein
A/G Sepharose overnight with rocking at $4^{\circ} \mathrm{C}$. After the incubation, immune complexes were washed twice with lysis buffer, twice with $1 \mathrm{X}$ PBS, and were then resuspended in Laemmeli's sample buffer and boiled for $5 \mathrm{~min}$. For immunoblotting and immunoprecipitation, samples were resolved on a sodium dodecyl sulfate (SDS)-10\% polyacrylamide gel. Then proteins were electrophoretically transferred to a Protran nitrocellulose transfer and immobilization membrane (Schleicher and Schuell, Dassel, Germany). The membrane was then blocked overnight at $4^{\circ} \mathrm{C}$ with BSA or milk in $1 \mathrm{X}$ TBS (10mM Tris- $\mathrm{HCl}[\mathrm{pH} 8.0], 150 \mathrm{mM} \mathrm{NaCl})$ and then probed with antibody overnight at $4{ }^{\circ} \mathrm{C}$ in $1 \mathrm{X}$ TBST buffer $(10 \mathrm{mM}$ Tris-HCl [pH 8.0], $150 \mathrm{mM} \mathrm{NaCl}, 0.05 \%$ Tween20). After three washes with $1 \mathrm{X}$ TBST, membranes were incubated for $1 \mathrm{hr}$ at $25^{\circ} \mathrm{C}$ with anti-mouse immunoglobulin (horseradish peroxidase-linked whole antibody from sheep) (Amersham Life Science, Inc., Arlington Heights, IL). The membrane was washed $5 \mathrm{X}$ in $1 \mathrm{X}$ TBST buffer, with 5 min intervals between buffer changes, and bound antibody was detected with enhanced luminol and oxidizing reagent as specified by the manufacturer (NEN Life Science Products, Boston, MA).

\section{Antibodies}

The following antibodies were purchased from Cell Signaling Technology (Danvers, MA): total AKT (rabbit, \#9272) and total p44/42 MAPK (Erk1/2) (3A7) (mouse, \#9107) were used at 1:1000. Anti-GAPDH (D16H-11) XP (R) (rabbit mAb, \#5174) was used at 1:1000. PhosphoAKT (Ser 473) (D9E) XP(R) (rabbit mAb, \#4060) was used at 1:1000. Phospho-p44/42 MAPK (T202/ Y204) (rabbit, \#9101) was used at 1:1000. Phospho-S6 ribosomal protein (S235/236)( D57.2.2E) XP (R) (rabbit $\mathrm{mAb}, \# 4858)$ was used at 1:1000. Total S6 ribosomal protein (SG10) (rabbit mAb, \#2217) was used at 1:1000. Phospho-STAT5 Tyr694 (rabbit, \#9351S) and total STAT5 (3H7) (rabbit, \#9358 mAb) were used at 1:1000. The Syk (D3Z1E) XP (rabbit mAb, \#13198) was used at 1:1000.

FLT3/Flk-2 (C-20) (sc-479) was purchased from Santa Cruz Biotechnology, Inc., (Dallas, TX) and used at 1:1000 for immunoblotting. Anti-pTyr (mouse, clone 4G10) was purchased from Upstate Biotechnology (Lake Placid, NY) and was used at 1:1000 in the presence of $4 \%$ BSA.

\section{Cell growth studies}

The trypan blue exclusion assay has been previously described [6] and was used for quantification of cells prior to seeding for Cell Titer Glo assays. The CellTiter Glo assay (Promega, Madison, WI) was used for proliferation studies and carried out according to manufacturer instructions. Cell viability is reported as percentage of 
control (untreated) cells, and error bars represent the standard deviation for each data point.

\section{Apoptosis assays and cell cycle analysis}

Programmed cell death of inhibitor-treated cells was determined using the Annexin-V-Fluos Staining Kit (Boehringer Mannheim, Indianapolis, IN), as previously described [6]. Briefly, cells were washed once with $1 \mathrm{X}$ PBS and pelleted by centrifugation for 5 minutes at 1500 rpm. Cells were resuspended in $100 \mu$ of $20 \%$ propidium iodide (PI) and 20\% Annexin-V-fluorescein labeling reagent, either agent alone (as controls), or were left unstained by diluting only in $1 \mathrm{X}$ binding buffer (as a control). All samples were incubated for 10-15 minutes at room temperature, and then stained cells were diluted in $0.8 \mathrm{~mL}$ of $1 \mathrm{X}$ binding buffer. Cells were then analyzed by flow cytometry.

Cell cycle analysis was performed as previously described [6]. Briefly, around 500,000 cells were centrifuged at $1500 \mathrm{rpm}$ for $5 \mathrm{~min}$ and washed in $1 \mathrm{X}$ PBS, and the pellet was resuspended in $500 \mu$ of propidium iodide solution $(50 \mu \mathrm{g} / \mathrm{ml}$ propidium iodide, $0.1 \% \mathrm{NP}-40$, $0.1 \%$ sodium citrate). The mixture was stored in the dark at $4^{\circ} \mathrm{C}$ for a minimum of $15 \mathrm{~min}$, and then analyzed by flow cytometry.

\section{Caspase 3 activation assay}

For measurement of caspase- 3 and -7 activity, the Apo-ONE Homogenous Caspase-3/7 Assay kit was used (Promega, Madison, WI). The assay was carried out according to manufacturer's instructions.

\section{Drug combination studies}

For drug combination studies, cell viability was first determined using the Trypan Blue exclusion assay to quantify cells for cell seeding, and Cell Titer Glo was then implemented for proliferation studies. Single agents were added simultaneously at fixed ratios to cells. Cell viability was expressed as the function of growth affected (FA) drug-treated versus control cells; data were analyzed by Calcusyn software (Biosoft, Ferguson, MO and Cambridge, UK), which was utilized for synergy measurement and based on isobologram generation and the method of Chou-Talalay (1984) [33]. This method utilizes the median effect principle to quantify the effects of drug combinations to determine whether they give greater effects together than expected from a simple summation of their individual effects. After determining the $\mathrm{ED}_{50}$ or $\mathrm{IC}_{50}$ of each drug, combinations are studied where the concentrations are multiples, or fractions, of the $\mathrm{ED} / \mathrm{IC}_{50}$. Statistical analysis is automatically part of the computations. Combination indices, values generated by the Calcusyn software, which are less than one indicate synergy, whereas values greater than one indicate antagonism. Calcusyn combination indices can be interpreted as follows: $\mathrm{CI}<0.1$ indicate very strong synergism; values $0.1-0.3$ indicate strong synergism; values 0.3-0.7 indicate synergism; values 0.7-0.85 indicate moderate synergism; values $0.85-0.90$ indicate slight synergism; values 0.9-1.1 indicate nearly additive effects; values 1.10-1.20 indicate slight antagonism; values 1.20-1.45 indicate moderate antagonism; values 1.453.3 indicate antagonism; values 3.3-10 indicate strong antagonism; values $>10$ indicate very strong antagonism.

\section{Author contributions}

Ellen L. Weisberg is responsible for generation of research findings reported in paper (design/ performance of experiments, including immunoblotting, immunoprecipitation, proliferation, and synergy studies), integrity and analysis of the data, writing of the manuscript.

Alex Puissant provided $\mathrm{Ba} / \mathrm{F} 3$ cell lines engineered to over-express active SYK and/or FLT3-ITD.

Richard Stone offered valuable scientific feedback and helped with conception of research reported in paper.

Martin Sattler offered valuable scientific feedback and helped with conception of research reported in paper.

Sara J. Buhrlage assisted with obtaining SelectScreen Kinase Profiling results for midostaurin.

Jing Yang performed densitometry.

Paul W. Manley offered valuable scientific feedback and helped with conception of research reported in paper.

Chengcheng Meng performed annexin/pi staining and cell cycle analysis.

Michael Buonopane assisted with annexin/pi and cell cycle analysis.

John F. Daley assisted with annexin/pi and cell cycle analysis.

Suzan Lazo assisted with annexin/pi and cell cycle analysis.

Renee Wright performed annexin/pi staining and cell cycle analysis.

David M. Weinstock supervised AML primagraft sample distribution.

Amanda L. Christie provided AML primagraft samples and patient information.

Kimberly Stegmaier provided $\mathrm{Ba} / \mathrm{F} 3$ cell lines engineered to over-express active SYK and/or FLT3-ITD, and offered valuable scientific feedback and helped with the conception of research reported in paper.

James D. Griffin offered valuable scientific feedback and helped with conception of research reported in paper. 


\section{CONFLICTS OF INTEREST}

P.W.M. is an employee of Novartis Pharma AG. J.D.G. and P.M. have a financial interest with Novartis Pharma AG.

\section{FUNDING}

This work was funded by the NIH grant P01CA66996.

\section{REFERENCES}

1. Cancer Genome Atlas Research Network. Genomic and epigenomic landscapes of adult de novo acute myeloid leukemia. N Engl J Med. 2013; 368:2059-2074.

2. Ofran Y, Rowe JM. Genetic profiling in acute myeloid leukaemia-Where are we and what is its role in patient management. Br J Haematol. 2013; 160:303-320.

3. Martelli M.P, Sportoletti P, Tiacci E, Martelli MF, Falini B. Mutational landscape of AML with normal cytogenetics: Biological and clinical implications. Blood Rev. 2013; 27:13-22.

4. Gallogly MM, Lazarus HM. Midostaurin: an emerging treatment for acute myeloid leukemia patients. J Blood Med. 2016; 7:73-83.

5. Weisberg E, Barrett R, Liu Q, Stone R, Gray N, Griffin JD. FLT3 inhibition and mechanisms of drug resistance in mutant FLT3-positive AML. Drug Resist Updat. 2009; 12:81-89.

6. Weisberg E, Boulton C, Kelly LM, Manley P, Fabbro D, Meyer T, Gilliland DG, Griffin JD. Inhibition of mutant FLT3 receptors in leukemia cells by the small molecule tyrosine kinase inhibitor PKC412. Cancer Cell. 2002; $1: 433-443$

7. Stone RM, Mandrekar S, Sanford BL, Geyer S, Bloomfield CD, Dohner K, Thiede C, Marcucci G, Lo-Coco F, Klisovic RB, Wei A, Sierra J, Sanz MA, et al. The multi-kinase inhibitor midostaurin $(\mathrm{M})$ prolongs survival compared with placebo $(\mathrm{P})$ in combination with daunorubicin (D)/ cytarabine (C) induction (ind), high-dose $\mathrm{C}$ consolidation (consol), and as maintenance (maint) therapy in newly diagnosed acute myeloid leukemia (AML) patients (pts) age 18-60 with FLT3 mutations (muts): An international prospective randomized (rand) P-controlled double-blind trial (CALGB 10603/RATIFY [Alliance]) (abstract); Paper presented at: American Society of Hematology (ASH) 57th Annual Meeting; December 3-6, 2015; Orlando, FL, USA.

8. Chao Q, Sprankle KG, Grotzfeld RM, Lai AG, Carter TA, Velasco AM, Gunawardane RN, Cramer MD, Gardner MF, James J, Zarrinkar PP, Patel HK, Bhagwat SS. Identification of N-(5-tert-butyl-isoxazol-3-yl)-N'-\{4-[7-(2-morpholin4-yl-ethoxy)imidazo[2,1-b][1,3]benzothiazol-2-yl]phenyl $\}$ urea dihydrochloride (AC220), a uniquely potent, selective, and efficacious FMS-like tyrosine kinase-3 (FLT3) inhibitor. J Med Chem. 2009; 52:7808-7816.

9. Smith CC, Lasater EA, Lin KC, Wang Q, McCreery MQ, Stewart WK, Damon LE, Perl, AE, Jeschke GR, Sugita M, Carroll M, Kogan SC, Kuriyan J, Shah NP. Crenolanib is a selective type I pan-FLT3 inhibitor. Proc Natl Acad Sci. USA 2014; 111:5319-5324.

10. Puissant A, Fenouille N, Alexe G, Pikman Y, Bassil CF, Mehta S, Du J, Kazi JU, Luciano F, Roonstrand L, Kung AL, Aster JC, Galinsky I, et al. SYK is a critical regulator of FLT3 in acute myeloid leukemia. Cancer Cell. 2014; $25: 226-42$

11. Turner M, Mee PJ, Costello PS, Williams O, Price AA, Duddy LP, Furlong MT, Geahlen RL, Tybulewicz VL. Perinatal lethality and blocked B-cell development in mice lacking the tyrosine kinase Syk. Nature. 1995; 378:298-302.

12. Cheng AM, Rowley B, Pao W, Hayday A, Bolen JB, Pawson T. Syk tyrosine kinase required for mouse viability and B-cell development. Nature. 1995; 378:303-306.

13. Bu JY, Shaw AS, Chan AC. Analysis of the interaction of ZAP-70 and syk protein-tyrosine kinases with the T-cell antigen receptor by plasmon resonance. Proc Natl Acad Sci USA. 1995; 92:5106-5110.

14. Cornall RJ, Cheng AM, Pawson T, Goodnow CC. Role of Syk in B-cell development and antigen-receptor signaling. Proc Natl Acad Sci USA. 2000; 97:1713-1718.

15. Rinaldi A, Kwee I, Taborelli M, Largo C, Uccella S, Martin V, Poretti G, Gaidano G, Calabrese G, Martinelli G, Baldini L, Pruneri G, Capella $\mathrm{C}$ et al. Genomic and expression profiling identifies the B-cell associated tyrosine kinase Syk as a possible therapeutic target in mantle cell lymphoma. $\mathrm{Br}$ J Haematol. 2006; 132:303-316.

16. Chen L, Monti S, Juszczynski P, Daley J, Chen W, Witzig TE, Habermann TM, Kutok JL, Shipp MA. SYK-dependent tonic B-cell receptor signaling is a rational treatment target in diffuse large B-cell lymphoma. Blood. 2008; 111:2230 2237.

17. Buchner M, Fuchs S, Prinz G, Pfeifer D, Bartholome K, Burger M, Chevalier N, Vallat L, Timmer J, Gribben JG, Jumaa H, Veelken H, Dierks C, et al. Spleen tyrosine kinase is overexpressed and represents a potential therapeutic target in chronic lymphocytic leukemia. Cancer Res. 2009; 69:5424-5432.

18. Hahn CK, Berchuck JE, Ross KN, Kakoza RM, Clauser K, Schinzel AC, Ross L, Galinsky I, Davis TN, Silver SJ, Root DE, Stone RM, DeAngelo DJ, et al. Proteomic and genetic approaches identify Syk as an AML target. Cancer Cell. 2009; 16:281-294.

19. Pechloff K, Holch J, Ferch U, Schweneker M, Brunner K, Kremer M, Sparwasser T, Quintanilla-Martinez L, ZimberStrobl U, Streubel B, Gewies A, Peschel C, Ruland J. The fusion kinase ITK-SYK mimics a $\mathrm{T}$ cell receptor signal and drives oncogenesis in conditional mouse models of peripheral T cell lymphoma. J Exp Med. 2010; 207:1031- 
1044.

20. Streubel B, Vinatzer U, Willheim M, Raderer M, Chott A. Novel $\mathrm{t}(5 ; 9)(\mathrm{q} 33 ; \mathrm{q} 22)$ fuses ITK to SYK in unspecified peripheral T-cell lymphoma. Leukemia. 2006; 20:313-318.

21. Kuno Y, Abe A, Emi N, Iida M, Yamamori T, Tanimoto M, Saito H. An atypical myelodysplastic syndrome with $\mathrm{t}(9 ; 12)(\mathrm{q} 22 ; \mathrm{p} 12)$ and TEL gene rearrangement. $\mathrm{Br} \mathrm{J}$ Haematol. 1999; 106:570-571.

22. Kuno Y, Abe A, Emi N, Iida M, Yokozawa T, Towatari M, Tanimoto M, Saito H. Constitutive kinase activation of the TEL-Syk fusion gene in myelodysplastic syndrome with $\mathrm{t}(9 ; 12)(\mathrm{q} 22 ; \mathrm{p} 12)$. Blood. 2001; 97:1050-1055.

23. Wossning T, Herzog S, Kohler F, Meixlsperger S, Kulathu Y, Mittler G, Abe A, Fuchs U, Borkhardt A, Jumaa H. Deregulated Syk inhibits differentiation and induces growth factor-independent proliferation of pre-B cells. J Exp Med. 2006; 203:2829-2840.

24. Sprissler C, Belenki D, Maurer H, Aumann K, Pfeifer D, Klein C, Muller TA, Kissel S, Hulsdunker J, Alexandrovski J, Brummer T, Jumaa H, Duyster J, et al. Depletion of STAT5 blocks TEL-SYK-induced APMF-type leukemia with myelofibrosis and myelodysplasia in mice. Blood Cancer J. 2014; 4:e240.

25. Peter B, Winter GE, Blatt K, Bennett KL, Stefanzl G, Rix U, Eisenwort G, Hadzijusufovic E, Gridling M, Dutreix C, Hoermann G, Schwaab J, Radia D, et al. Target interaction profiling of midostaurin and its metabolites in neoplastic mast cells predicts distinct effects on activation and growth. Leukemia. 2016; 30:464-72.

26. Braselmann S, Taylor V, Zhao H, Wang S, Sylvain C, Baluom M, Qu K, Herlaar E, Lau A, Young C, Wong BR, Lovell S, Sun T, et al. R406, an orally available spleen tyrosine kinase inhibitor blocks fc receptor signaling and reduces immune complex-mediated inflammation. J Pharmacol Exp Ther. 2006; 319:998-1008.
27. Coffey G, DeGuzman F, Inagaki M, Pak Y, Delaney SM, Ives D, Betz A, Jia ZJ, Pandey A, Baker D, Hollenbach SJ, Phillips DR, Sinha U. Specific inhibition of spleen tyrosine kinase suppresses leukocyte immune function and inflammation in animal models of rheumatoid arthritis. J Pharmacol Exp Ther. 2012; 340:350-9.

28. Weisberg E, Ray A, Nelson E, Adamia S, Barrett R, Sattler M, Zhang C, Daley JF, Frank D, Fox E, Griffin JD. Reversible resistance induced by FLT3 inhibition: a novel resistance mechanism in mutant FLT3-expressing cells. PLoS One. 2011; 6:e25351.

29. Beitz LO, Fruman DA, Kurosaki T, Cantley LC, Scharenberg AM. SYK is upstream of phosphoinositide 3-kinase in B cell receptor signaling. J Biol Chem. 1999; 274:32662-32666.

30. Chiu CW, Dalton M, Ishiai M, Kurosaki T, Chan AC. BLNK: molecular scaffolding through, cis'-mediated organization of signaling proteins. EMBO J. 2002; 21:64616472.

31. Matsuo Y, MacLeod RA, Uphoff CC, Drexler HG, Nishizaki C, Katayama Y, Kimura G, Fujii N, Omoto E, Harada M, Orita K. Two acute monocytic leukemia (AMLM5a) cell lines (MOLM13 and MOLM14) with interclonal phenotypic heterogeneity showing MLL-AF9 fusion resulting from an occult chromosome insertion, ins(11;9) (q23;p22p23). Leukemia. 1997; 11:1469-77.

32. Armstong SA, Kung AL, Mabon ME, Silverman LB, Stam RW, Den Boer ML, Pieters R, Kersey JH, Sallan SE, Fletcher JA, Golub TR, Griffin JD, Korsmeyer SJ. Validation of a therapeutic target identified by gene expression based classification. Cancer Cell. 2003; 3:17383.

33. Chou TC, Talalay P. Quantitative analysis of dose-effect relationships: the combined effects of multiple drugs or enzyme inhibitors. Adv Enzyme Regul. 1984; 22:27-55. 